\title{
THE MIDDLE MIOCENE CENTRAL EUROPEAN PLANT RECORD REVISITED; WIDESPREAD SUBHUMID SCLEROPHYLLOUS FORESTS INDICATED
}

\author{
JOHANNA KOVAR-EDER ${ }^{1, *}$, VASILIS TEODORIDIS ${ }^{2}$ \\ 1 State Museum of Natural History Stuttgart, Rosenstein 1, 70191 Stuttgart, Germany; e-mail: johanna.eder@smns-bw.de. \\ 2 Department of Biology and Environmental Studies, Faculty of Education, Charles University, Magdalény Rettigové 4, 11639 Prague 1, \\ the Czech Republic; e-mail: vasilis.teodoridis@pedf.cuni.cz. \\ * corresponding author
}

Kovar-Eder, J., Teodoridis, V. (2018): The Middle Miocene Central European plant record revisited; widespread subhumid sclerophyllous forests indicated. - Fossil Imprint, 74(1-2): 115-134, Praha. ISSN 2533-4050 (print), ISSN $2533-4069$ (on-line).

\begin{abstract}
The Middle Miocene plant record from the wider Central Paratethys region (Central Paratethys, the North Alpine Foredeep, Carpathian Foredeep, the Swabian Alb and southern parts of the Bohemian Massif) was analysed by the Integrated Plant Record (IPR) vegetation analysis to assess major vegetation types. The plant assemblages at 27 sites accumulated under very different sedimentological settings and reflect heterogeneous environments. Although of very different origin, the plant record delivers a fairly consistent signal towards subhumid sclerophyllous forests (ShSF) as the most likely major zonal vegetation unit for the Langhian/Serravallian. Today, such forests develop under seasonal climate with pronounced seasonal changes in precipitation. The floristic characteristics are outlined and the record is compared to that of the preceding and the following Miocene periods.
\end{abstract}

Key words: zonal vegetation, Integrated Plant Record vegetation analysis, Middle Miocene, Central Europe

Received: February 20, 2018 | Accepted: April 23, 2018 | Issued: August 31, 2018

\section{Introduction}

The Middle Miocene, currently dated to $15.97-11.63$ m.a. (Cohen et al. 2013 [updated 2017]), was a period of severe abiotic and biotic changes both on a global and regional scale. Two events partly fall into this period: the Mid-Miocene Climatic Optimum (approximately 17-15 m.a.) as well as the Middle Miocene Climate Transition at 14.2 to 13.8 m.a., which is linked to the expansion of the Antarctic ice sheet (Shevenell et al. 2004). The Central Paratethys region underwent several transgressional cycles, a severe sea-level drop of up to 120 $\mathrm{m}$ probably related to the afore-mentioned growth of the Antarctic ice shield, and the demise of marine conditions in the North Alpine Foredeep during the Badenian (Harzhauser and Piller 2007).

The fossil plant record of this period has been interpreted more controversially than for any other Miocene period ranging from "Buschsteppe", "Savanne" and "Macchia- und Hartlaubgewächse mediterraner Prägung" (Berger and Zabusch 1953) and Mediterranean-like vegetation (Andreánszky 1959, Szafer 1961) to deciduous mesophytic forests under (semi) humid-temperate climate (Cfa sensu Köppen) for plant assemblages from the Alpine Foredeep (Gregor 1980, 1982).

For the Late Badenian and Middle Pannonian, Kvaček et al. (2006) traced the vegetation of the Paratethys region on palinspastic palaeogeographic maps, thus only partly covering the here-discussed region and time interval. Further studies considered whole western Eurasia. These include the vegetation assessments performed by KovarEder and Kvaček (2007) and Kovar-Eder et al. (2008) based on the Integrated Plant Record (IPR) vegetation analysis and by Utescher et al. (2007) evaluating plant functional types. Most recently, Henrot et al. (2017) deduced the vegetation from climate models for the Middle Miocene.

Attempts to assess climatic evolution are based on diverse resources such as herpetofauna (e.g. Böhme 2003, Böhme et al. 2008, 2011), mammals (e.g. Van Dam and Utescher 2016) and plants (Erdei et al. 2007, Bruch et al. 2011). The results of those studies are partly also very controversial, assuming distinctly drier conditions in the Langhian/Serravallian compared to the earlier and later periods (Böhme et al. 2011), while concluding very high precipitation in the Langhian and lower precipitation with seasonal changes in the Serravallian (Bruch et al. 2011). Other attempts are too imprecise to decipher regional features (e.g. Henrot et al. 2017). Here, we reinvestigate the rich Middle Miocene plant record of the wider Central Paratethys region by applying the IPR vegetation analysis to assess zonal vegetation units, i.e. vegetation at low altitudes under mesic conditions. 


\section{Material and methods}

From the Langhian/Serravallian, 31 plant assemblages (leaves, fruits, pollen) from 27 sites were selected from the wider Central Paratethys region. This includes the Central Paratethys, the Carpathian and the North Alpine Foredeeps, the Norian Depression, the Swabian Alb, and the southern Bohemian Massif (Text-figs 1, 2, Tab. 1). Strong emphasis is put on independent dating (i.e. dating by means other than by plant remains) to achieve reliable resolution in time. The depositional facies range from maar lake deposits at Randeck Maar and lacustrine sediments in the impact crater lake at Steinheim on the Swabian Alb to lacustrine sediments (Parschlug, Weingraben) and fluvio-lacustrine deposits (e.g. Kirrberg, Entrischenbrunn, Schrotzburg, Młyny and Stawiany) as well as marine/brackish (e.g. Wieliczka, Erdőbénye, Türkenschanze, Hernals) and floodplain settings (Gratkorn). Some sites were previously examined applying the IPR vegetation analysis when the time interval 14-17 m.a. was studied (Kovar-Eder and Kvaček 2007, KovarEder et al. 2008). Such sites are included here again due to the modified spatio-temporal focus of this study.

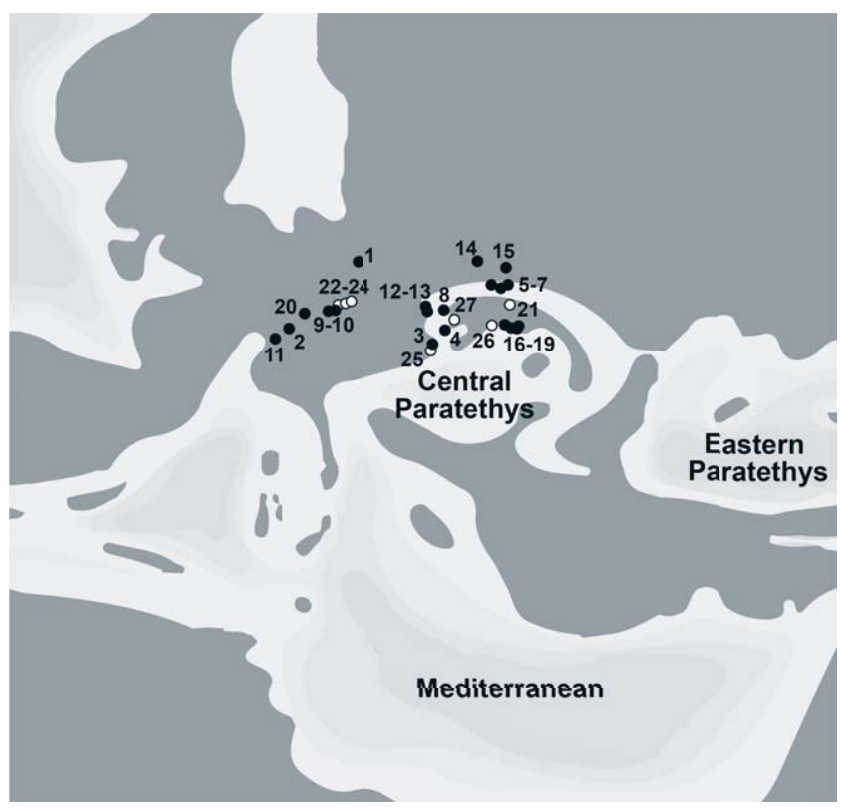

Text-fig. 1. Palaeogeographic map of the Langhian/Badenian according to Harzhauser and Piller (2007) and location of the fossil-bearing sites. Numbers of sites correspond to those in Table 1. Black circles - IPR vegetation analysis applied, white circles - IPR vegetation analysis not applicable.

\section{The Integrated Plant Record (IPR) vegetation analysis}

This method was developed to assess major vegetation types. It applies a semi-quantitative evaluation based on the autecology of taxa and leaf physiognomy (Kovar-Eder and Kvaček 2003, 2007, Kovar-Eder et al. 2008, Teodoridis et al. 2011a, b). The fossil plant record is usually strongly influenced or even dominated by intrazonal taxa (mainly wetland taxa), masking zonal taxa which are characteristic of mesophytic environments. The fossil flora of Parschlug serves as a showcase in this respect, where a few wetland taxa are most abundant and mask the true nature of this assemblage (Kovar-Eder et al. 2004). A major feature of the IPR vegetation analysis is, therefore, to exclude intrazonal taxa to the degree possible to uncover the true composition of the mesophytic vegetation. This is important because the latter is essential for extracting climate signals.

In performing the IPR vegetation analysis, all taxa of an assemblage are assigned to the components described in detail in Teodoridis et al. (2011a). The major vegetation types are assessed by the percentages of broad-leaved deciduous (BLD), broad-leaved-evergreen (BLE) and sclerophyllous plus legume-like (SCL+LEG) components of zonal angiosperm taxa. We are aware that the assessment of some taxa (e.g. Quercus kubinyii (KovÁts ex EtTingsh.) Czeczott) may be equivocal and autecology of fossil taxa may differ from the most similar living relatives. Since the reliability of the results depends on the number of zonal taxa available in an assemblage, here we raise the former threshold from a minimum of 10 zonal taxa (Kovar-Eder et al. 2008) to 15 for the application of the IPR vegetation analysis. The major vegetation types as defined for the IPR vegetation analysis are illustrated in Table 2 . Refining the thresholds of components (Teodoridis et al. 2011a) caused slight differences compared to the evaluation results presented earlier by Kovar-Eder and Kvaček (2007) and Kovar-Eder et al. (2008). Taxonomic updates were made prior to the IPR vegetation analysis (Appendix 1).

\section{Cluster analysis}

A hierarchical tree clustering analysis was performed applying the STATGRAPHICS program in which the fossil record is clustered in the context of modern vegetation units from China and Japan (Teodoridis et al. 2011a, 2012). Ward's method was applied as a linkage tree clustering method in which two clusters $(\mathrm{x}, \mathrm{y})$ are determined by the analysis of variance, and the method joins the clusters with minimal sums of squares (the Euclidean square distance). The number of the defaulted clusters was one. The percentages of the BLD, BLE and SCL+LEG components of the studied fossil and modern sites derived by the IPR vegetation analysis served as a source for cluster analysis (Appendix 2). The dendrogram shows the relationships between modern vegetation from tropical, subtropical and temperate zones of SE China and Japan (Teodoridis et al. 2011a, Teodoridis et al. 2012) and of the here-studied fossil floras (Text-fig. 3).

Abbreviations: IPR vegetation analysis - Integrated Plant Record vegetation analysis, BLD component - broad-leaved deciduous component, BLE component - broad-leaved evergreen component, SCL component - sclerophyllous component, LEG component - legume-type component, BLDF - broad-leaved deciduous forest, BLEF - broadleaved evergreen forest, MMF - mixed mesophytic forest, ShSF - subhumid sclerophyllous forest.

\section{Results}

Twenty of 27 sites (24 assemblages of fruits, leaves, pollen) are suitable for the IPR vegetation analysis (Tab. 1). 


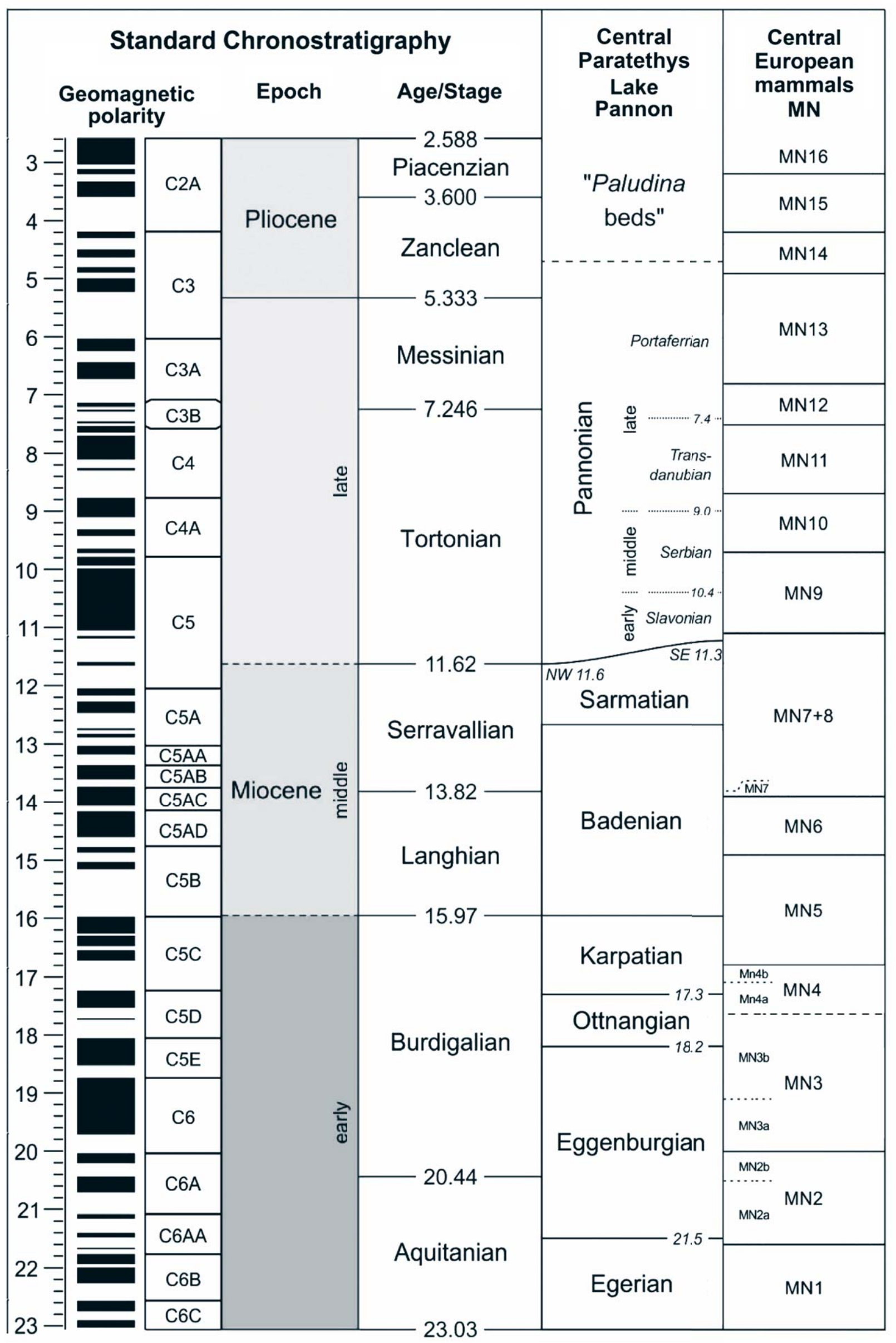

Text-fig. 2. Stratigraphic correlation table for the Miocene and Pliocene according to Neubauer et al. (2015). 


\begin{tabular}{|c|c|c|c|c|c|c|c|c|c|c|c|c|c|}
\hline 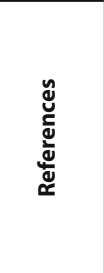 & 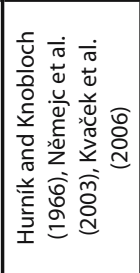 & 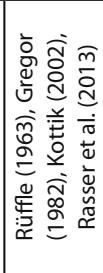 & 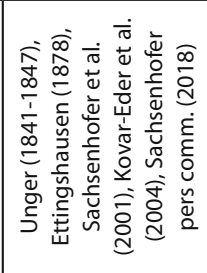 & 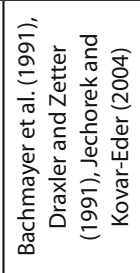 & 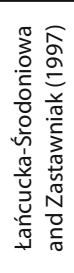 & 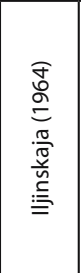 & 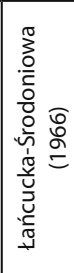 & 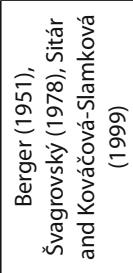 & 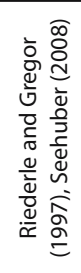 & 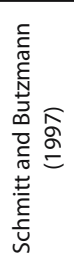 & 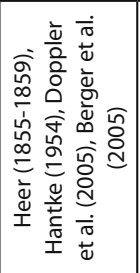 & 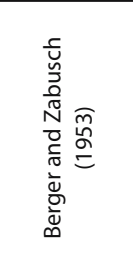 & 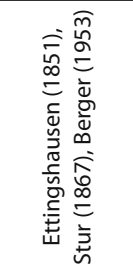 \\
\hline 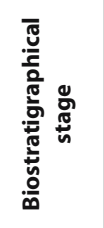 & 1 & 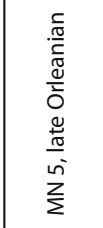 & 1 & 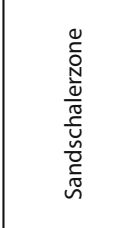 & 1 & 1 & 1 & 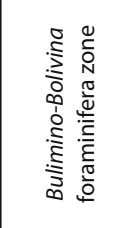 & 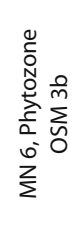 & 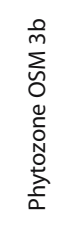 & 1 & 1 & 1 \\
\hline 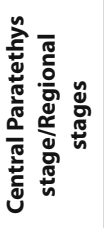 & 1 & 1 & 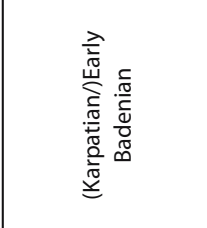 & 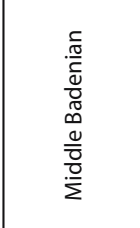 & 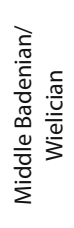 & 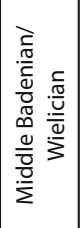 & 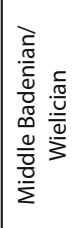 & 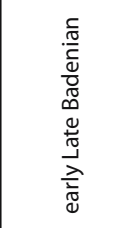 & 1 & 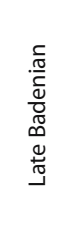 & 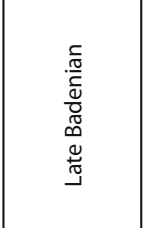 & 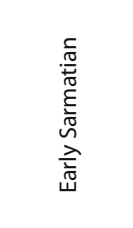 & 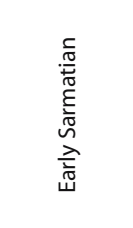 \\
\hline 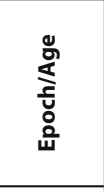 & 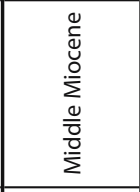 & 1 & 1 & 1 & 1 & 1 & 1 & 1 & 1 & 1 & 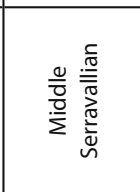 & 1 & 1 \\
\hline 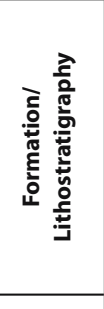 & 1 & 1 & 1 & 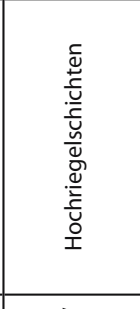 & 1 & 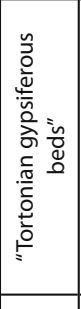 & 1 & 1 & 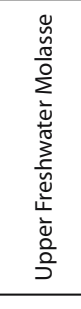 & 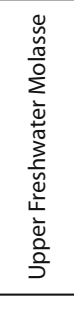 & 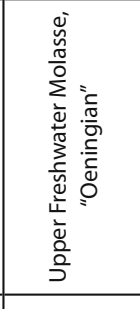 & 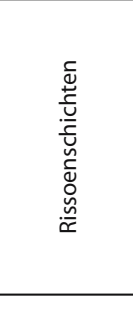 & 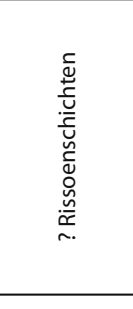 \\
\hline 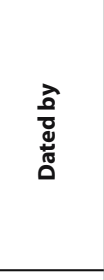 & $\stackrel{\frac{\pi}{0}}{\frac{0}{6}}$ & 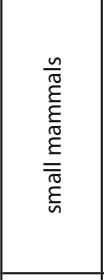 & 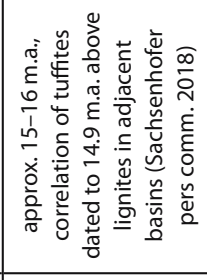 & 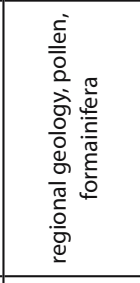 & 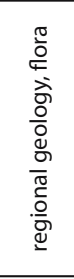 & 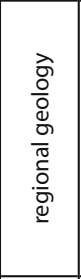 & 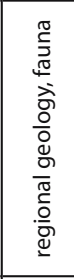 & 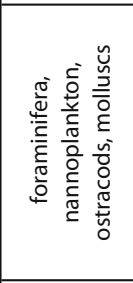 & 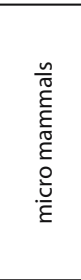 & $\frac{\underline{0}}{\mathrm{O}}$ & 1 & 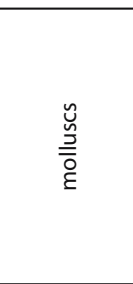 & 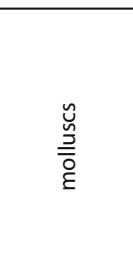 \\
\hline 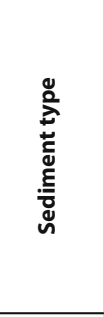 & 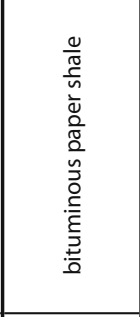 & 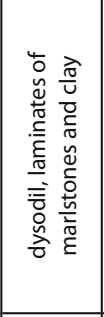 & 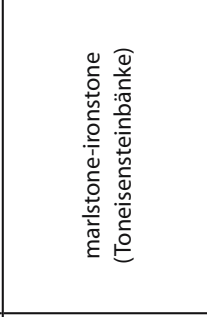 & $\begin{array}{l}\frac{\mathscr{u}}{\tilde{\pi}} \\
\frac{\tilde{m}}{\overline{\overline{0}}}\end{array}$ & 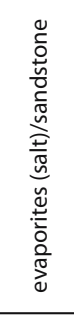 & 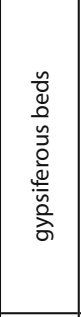 & 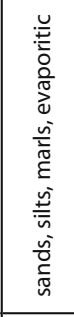 & $\frac{\text { ते }}{\mathrm{v}}$ & 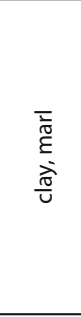 & $\stackrel{\frac{n}{\underline{m}}}{\stackrel{n}{\varepsilon}}$ & $\stackrel{\frac{n}{\tilde{m}}}{\stackrel{n}{E}}$ & $\frac{\text { ते }}{\mathrm{v}}$ & 1 \\
\hline 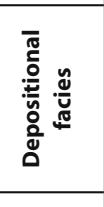 & 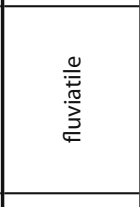 & 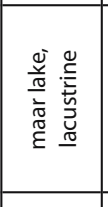 & 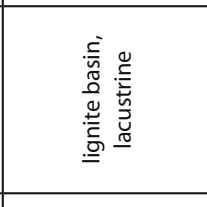 & 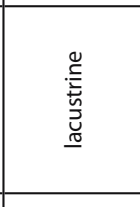 & 营 & . & 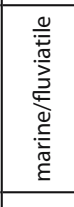 & 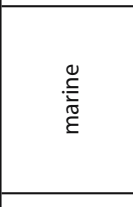 & 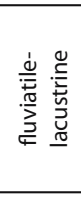 & 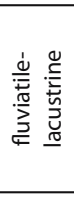 & 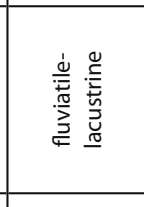 & 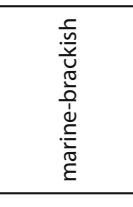 & 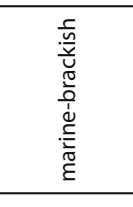 \\
\hline $\overrightarrow{\underline{\underline{E}}}$ & 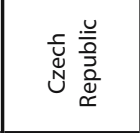 & $\begin{array}{l}\text { ते } \\
\text { हूँ } \\
\text { ज़ }\end{array}$ & 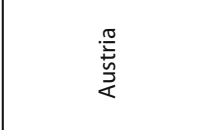 & 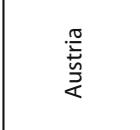 & 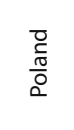 & 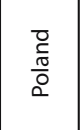 & $\begin{array}{l}\text { 뭉 } \\
\frac{5}{0} \\
\frac{0}{0}\end{array}$ & $\begin{array}{l}\frac{\pi}{\frac{\pi}{5}} \\
\text { 흔 }\end{array}$ & 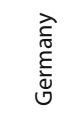 & $\begin{array}{l}\text { त्रे } \\
\text { हूँ } \\
\text { ज़ }\end{array}$ & 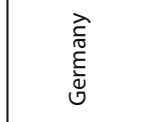 & 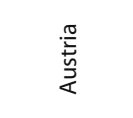 & 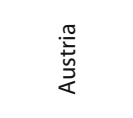 \\
\hline 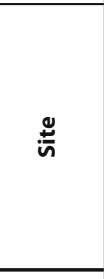 & 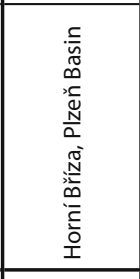 & 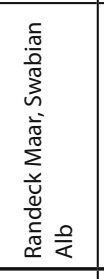 & 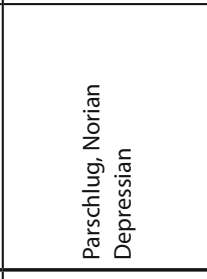 & 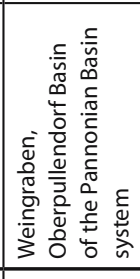 & 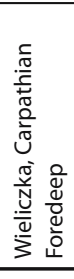 & 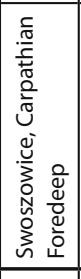 & 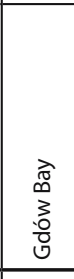 & 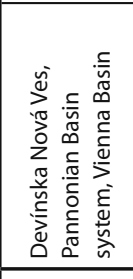 & 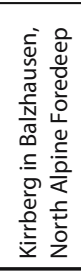 & 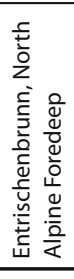 & 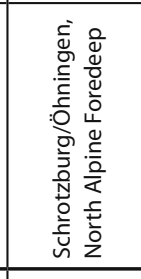 & 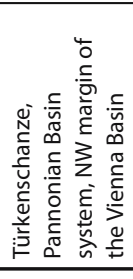 & 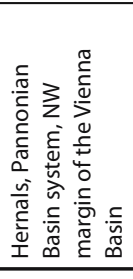 \\
\hline 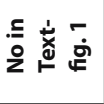 & - & $N$ & m & $\sigma$ & in & 0 & n & $\infty$ & $a$ & 음 & $=$ & $\simeq$ & $\underline{m}$ \\
\hline
\end{tabular}




\begin{tabular}{|c|c|c|c|c|c|c|c|c|c|c|c|c|c|c|c|}
\hline 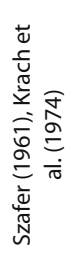 & \multicolumn{2}{|r|}{ 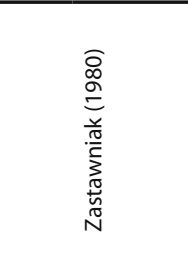 } & \multicolumn{4}{|c|}{ 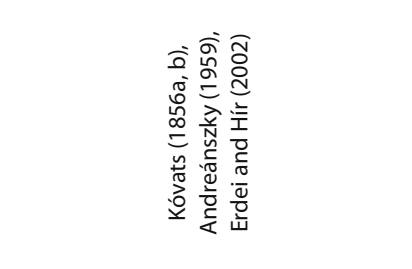 } & 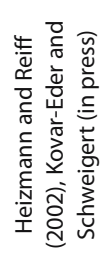 & & 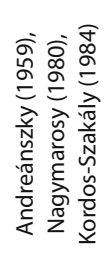 & 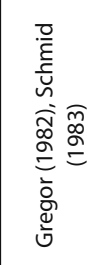 & 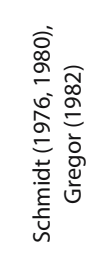 & 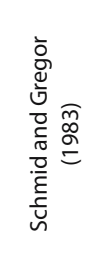 & 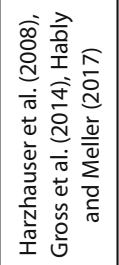 & 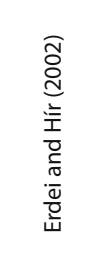 & 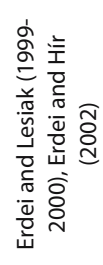 \\
\hline 1 & I & 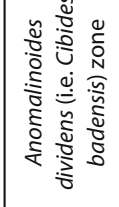 & 1 & 1 & 1 & 1 & $\frac{\infty}{\hat{\lambda}}$ & & $\sum_{z}^{n}$ & 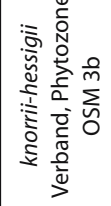 & 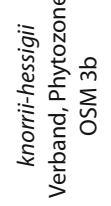 & 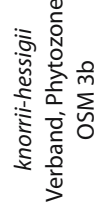 & 1 & $\sum_{\Sigma}^{\infty}$ & I \\
\hline 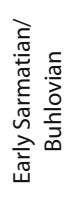 & 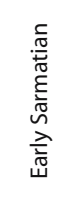 & 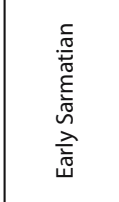 & 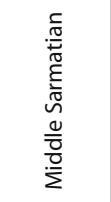 & 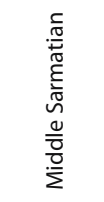 & 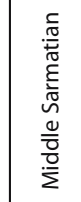 & 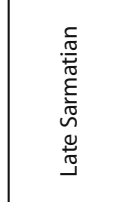 & 1 & & 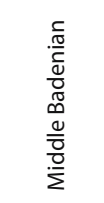 & 1 & 1 & 1 & 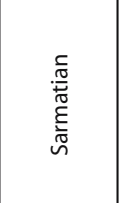 & 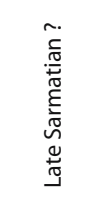 & 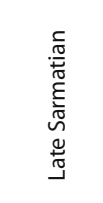 \\
\hline 1 & 1 & 1 & 1 & 1 & 1 & 1 & 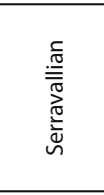 & 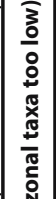 & 1 & 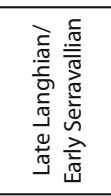 & 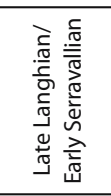 & 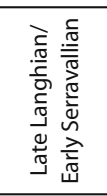 & 1 & 1 & 1 \\
\hline 1 & 1 & 1 & 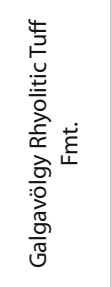 & 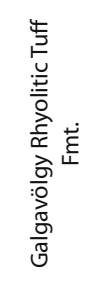 & 1 & 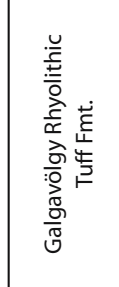 & 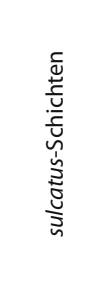 & 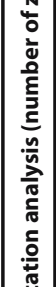 & 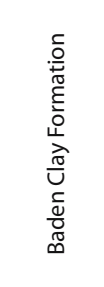 & 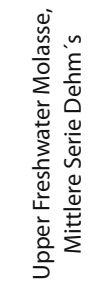 & 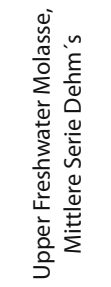 & 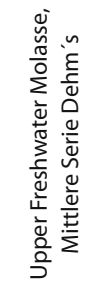 & 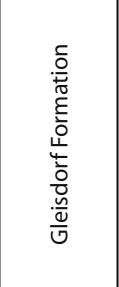 & ' & I \\
\hline 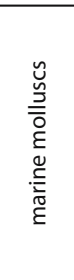 & 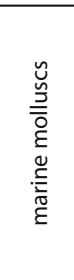 & 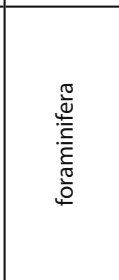 & 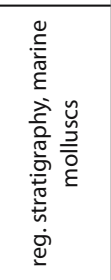 & 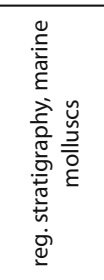 & 1 & 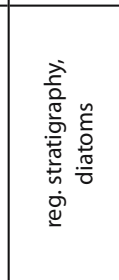 & 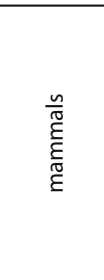 & 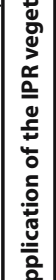 & 1 & 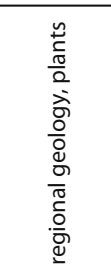 & 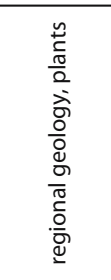 & 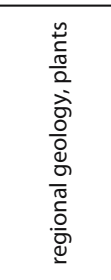 & 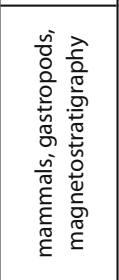 & 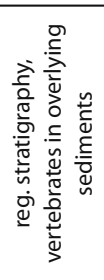 & 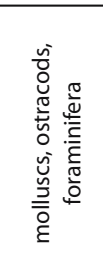 \\
\hline$\frac{\vec{\pi}}{\mathrm{v}}$ & 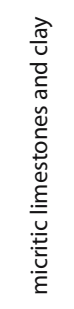 & 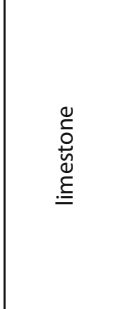 & 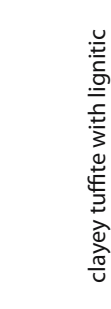 & & 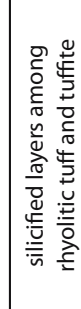 & 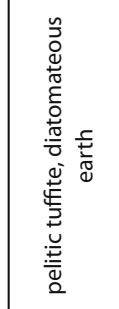 & 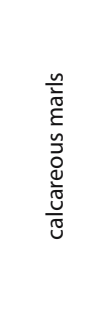 & 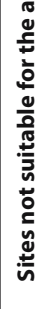 & 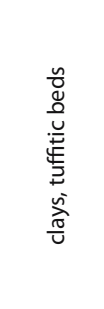 & I & 1 & 1 & $\frac{\widehat{\sigma}}{\mathrm{U}}$ & 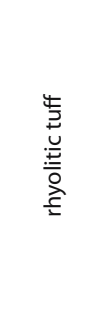 & 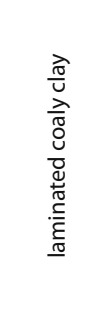 \\
\hline 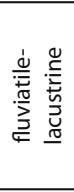 & 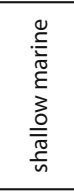 & 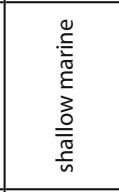 & 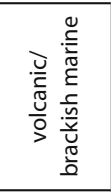 & 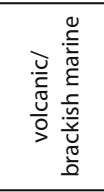 & $\begin{array}{l}\frac{\breve{\bar{E}}}{\overline{\mathrm{J}}} \\
\end{array}$ & 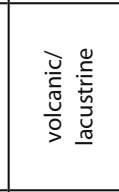 & 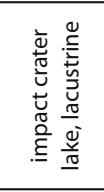 & & 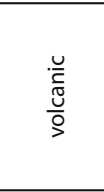 & 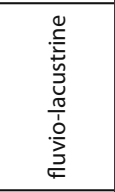 & 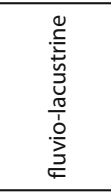 & 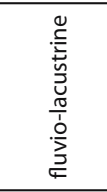 & 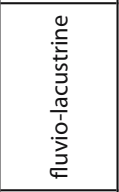 & 1 & 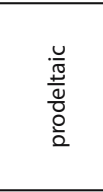 \\
\hline 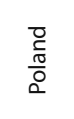 & $\begin{array}{l}\frac{7}{0} \\
\frac{\mathrm{c}}{0} \\
\frac{0}{0}\end{array}$ & $\begin{array}{l}\frac{\bar{c}}{\mathrm{c}} \\
\frac{\mathrm{T}}{0}\end{array}$ & 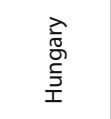 & 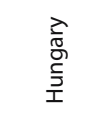 & 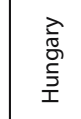 & 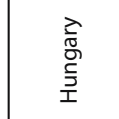 & 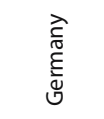 & & 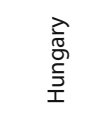 & 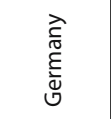 & $\begin{array}{l}\text { ते } \\
\text { हूँ } \\
\text { ভ. }\end{array}$ & 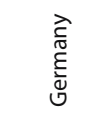 & 竞 & 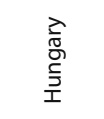 & 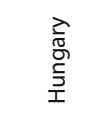 \\
\hline 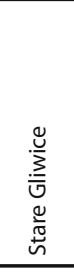 & 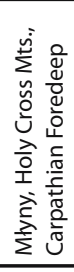 & 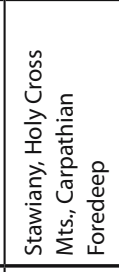 & 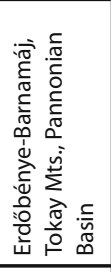 & 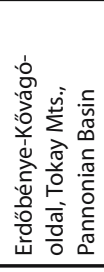 & 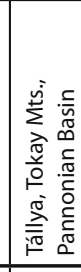 & 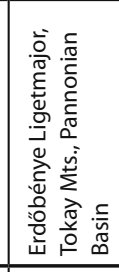 & 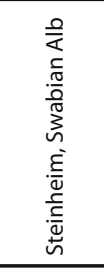 & & 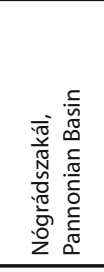 & 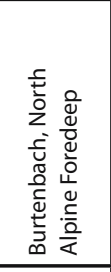 & 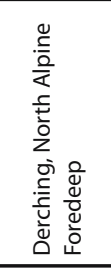 & 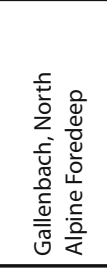 & 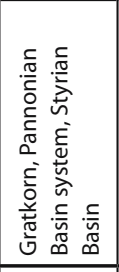 & 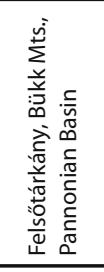 & 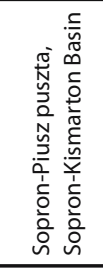 \\
\hline \pm & $\stackrel{n}{n}$ & $\stackrel{n}{n}$ & $\stackrel{0}{\circ}$ & 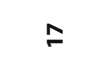 & $\stackrel{\infty}{-}$ & $\stackrel{a}{-}$ & i & & $\bar{N}$ & $\tilde{N}$ & $\tilde{\sim}$ & $\stackrel{\Delta}{\text { N }}$ & n & $\stackrel{\circ}{\sim}$ & $\hat{N}$ \\
\hline
\end{tabular}


Table 2. Zonal vegetation types as defined by the IPR vegetation analysis sensu Teodoridis et al. (2011a: tab. 8).

\begin{tabular}{|c|c|c|c|c|c|}
\hline \multirow[t]{2}{*}{ Vegetation type } & \multicolumn{3}{|c|}{ Zonal woody components } & \multirow{2}{*}{\begin{tabular}{|c|}
$\begin{array}{c}\text { Zonal herbaceous components } \\
\text { (fossil record) }\end{array}$ \\
MESO + DRY HERB \\
\end{tabular}} & \multirow{2}{*}{$\begin{array}{c}\begin{array}{c}\text { Zonal herbaceous } \\
\text { components (modern record) }\end{array} \\
\text { MESO + DRY HERB }\end{array}$} \\
\hline & BLD & BLE & SCL + LEG & & \\
\hline Broad-leaved decidous forests & $>80 \%$ & - & - & $\leq 30 \%$ & $40-70 \%$ \\
\hline Ecotone & $75-80 \%$ & 2000 & \multirow{3}{*}{$<20 \%$} & \multirow{3}{*}{$<30 \%$} & \multirow{3}{*}{$40-55 \%$} \\
\hline Mixed mesophytic forests & \multirow{2}{*}{$<80 \%$} & $<30 \%$ & & & \\
\hline Ecotone & & $30-40 \%$ & & & \\
\hline Broad-leaved evergreen forests & - & $>40 \%$ & $(\mathrm{SCL}+\mathrm{LEG})<\mathrm{BLE}$ & $<25 \%$ & $10-45 \%$ \\
\hline Subhumid sclerophyllous forests & - & & $\geq 20 \%$ & $<30 \%$ & $40-55 \%$ \\
\hline Xeric open woodlands & - & $<30 \%$ & $\geq 20 \%$ & $\begin{array}{c}30-40 \% \text {; MESO HERB }>\text { DRY } \\
\text { HERB up to } 10 \% \text { of all zonal } \\
\text { herbs }\end{array}$ & n.a. \\
\hline Xeric grasslands or steppe & - & $<30 \%$ & - & $\geq 40 \%$ & n.a. \\
\hline
\end{tabular}

Table 3. Summary of scoring results. Note that for sites included in previous studies (Kovar-Eder and Kvaček 2007, Kovar-Eder et al. 2008), some results have slightly changed due to refining the thresholds between the vegetation units (Teodoridis et al. 2011a).

\begin{tabular}{|c|c|c|c|c|c|c|c|c|c|}
\hline Site/Asemablage & BLD & BLE & SCL+LEG & Zonal palms & $\begin{array}{c}\text { Number of } \\
\text { taxa }\end{array}$ & $\begin{array}{c}\text { Number of } \\
\text { zonal woody } \\
\text { angiosperms }\end{array}$ & $\begin{array}{c}1 \text { taxon } \\
\text { accounts for } \\
\text { approx. } X \%\end{array}$ & Test & Vegetation type \\
\hline Horní Bříza & 62 & 15 & 20 & 4 & 32 & 24 & 4.2 & 100 & MMF/ShSF \\
\hline Randeck Maar-leaves & 40 & 28 & 26 & 6 & 49 & 33 & 3.1 & 100 & ShSF \\
\hline Randeck Maar-fruits & 65 & 11 & 24 & 0 & 47 & 20 & 4.9 & 100 & ShSF \\
\hline Randeck Maar-pollen & 48 & 35 & 15 & 2 & 54 & 24 & 4.2 & 100 & MMF/BLEF \\
\hline Randeck Maar integrated record & 49 & 26 & 22 & 3 & 150 & 77 & 1.3 & 100 & ShSF \\
\hline Parschlug & 48 & 18 & 34 & 0 & 74 & 54 & 1.9 & 100 & ShSF \\
\hline Weingraben-leaves-fruits & 37 & 26 & 37 & 0 & 37 & 29 & 3.5 & 100 & ShSF \\
\hline Weingraben-pollen & 57 & 31 & 12 & 0 & 57 & 23 & 4.4 & 100 & MMF/BLEF \\
\hline Weingraben integrated record & 46 & 28 & 26 & 0 & 94 & 52 & 1.9 & 100 & ShSF \\
\hline Wieliczka & 57 & 34 & 9 & 0 & 170 & 101 & 1.0 & 100 & MMF/BLEF \\
\hline Swoszowice & 78 & 9 & 12 & 0 & 27 & 16 & 6.2 & 100 & BLDF/MMF \\
\hline Gdów Bay & 86 & 9 & 5 & 0 & 58 & 20 & 5.1 & 158 & $\begin{array}{l}\text { BLDF but }>30 \% \\
\text { Meso+Dry herb }\end{array}$ \\
\hline Devínska Nová Ves & 62 & 27 & 11 & 0 & 78 & 36 & 2.8 & 100 & MMF \\
\hline Kirrberg & 62 & 22 & 16 & 0 & 40 & 27 & 3.7 & 100 & MMF \\
\hline Entrischenbrunn & 51 & 15 & 34 & 0 & 20 & 15 & 6.5 & 100 & ShSF \\
\hline Schrotzburg & 58 & 18 & 25 & 0 & 43 & 26 & 3.9 & 100 & ShSF \\
\hline Türkenschanze & 30 & 12 & 58 & 0 & 55 & 38 & 2.6 & 100 & ShSF \\
\hline Hernals & 34 & 29 & 37 & 0 & 27 & 19 & 5.2 & 100 & ShSF \\
\hline Stare Gliwice & 77 & 9 & 13 & 1 & 150 & 63 & 1.6 & 100 & MMF/BLDF \\
\hline Młyny & 48 & 5 & 47 & 0 & 52 & 31 & 3.3 & 100 & ShSF \\
\hline Młyny and Stawiany & 51 & 5 & 44 & 0 & 55 & 32 & 3.1 & 100 & ShSF \\
\hline Erdőbénye Barnamáj & 55 & 9 & 36 & 0 & 31 & 21 & 4.7 & 100 & ShSF \\
\hline Erdőbénye Kővágó-oldal & 55 & 10 & 35 & 0 & 43 & 30 & 3.3 & 100 & ShSF \\
\hline Erdőbénye Ligetmajor & 57 & 14 & 26 & 4 & 29 & 29 & 3.5 & 100 & ShSF \\
\hline Tállya & 68 & 4 & 28 & 0 & 30 & 24 & 4.1 & 100 & ShSF \\
\hline Steinheim & 61 & 6 & 32 & 0 & 44 & 28 & 3.5 & 100 & ShSF \\
\hline
\end{tabular}

The total number of all taxa per site varies between 20 (Entrischenbrunn) and 170 (Wieliczka) (Tab. 3). The number of zonal woody angiosperms is lowest in Entrischenbrunn (15) and highest in Wieliczka (101). In calculating the different components, a single taxon accounts for about $6.5 \%$ in the least confident calculation (Entrischenbrunn) to $1.0 \%$ in the most reliable results (Wieliczka). The BLD component varies between $30 \%$ (Türkenschanze) and $78 \%$ (Swoscowice). The BLE component is lowest in Tállya (4 \%) and highest in Randeck Maar-pollen (35\%); mostly it does not exceed $30 \%$. Exceptions are Randeck Maar-pollen, Weingraben-pollen, and Wieliczka. At 15 sites (17 assemblages), the values of the BLE component are lower than that of the SCL+LEG component. This involves most sites correlated to the Sarmatian. The value of the SCL+LEG component is lowest for Gdów Bay $(5 \%)$ and highest for Türkenschanze $(58 \%)$. At 6 sites $(8$ assemblages) this component accounts for $<20 \%$, which is the threshold towards ShSF according to the IPR vegetation analysis. These sites/assemblages are Randeck Maar-pollen, Weingraben-pollen, Wieliczka, Swoszowice, Gdów Bay, Devínska Nová Ves, Kirrberg and Stare Gliwice. At Horní Brriza the SCL+LEG component makes up $20 \%$. At all other sites (assemblages) the SCL+LEG component significantly exceeds the $20 \%$ threshold.

The IPR vegetation analysis yields a signal directed towards ShSF as the most likely major zonal vegetation unit for most sites (14 sites/15 assemblages). For the other 


\begin{tabular}{|c|c|c|c|c|c|c|c|c|c|c|}
\hline 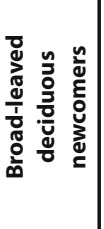 & 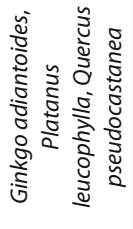 & 1 & 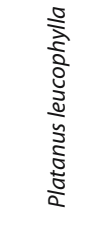 & 1 & 1 & 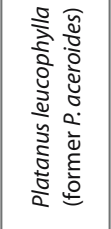 & 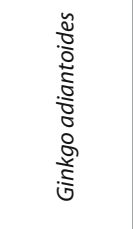 & 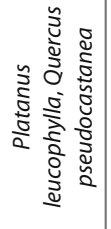 & 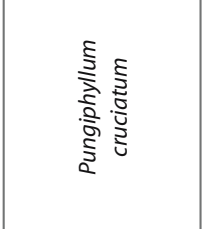 & 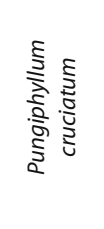 \\
\hline 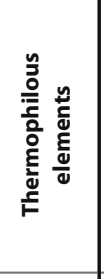 & 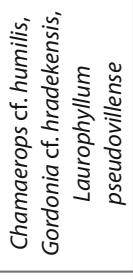 & 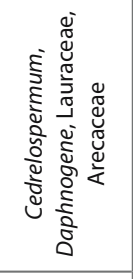 & 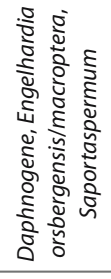 & 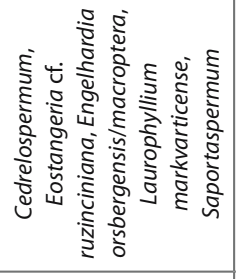 & 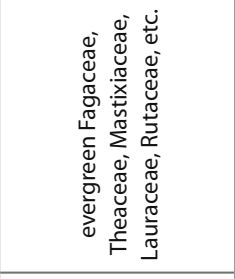 & 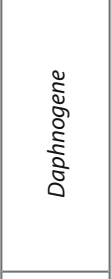 & 1 & 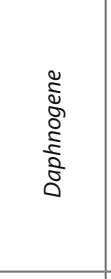 & 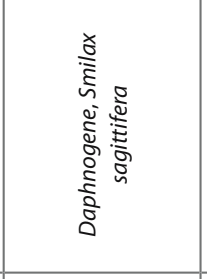 & 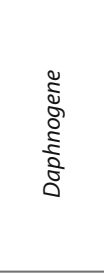 \\
\hline 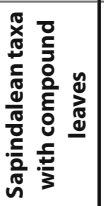 & 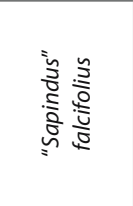 & 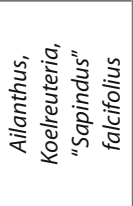 & 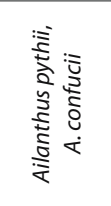 & 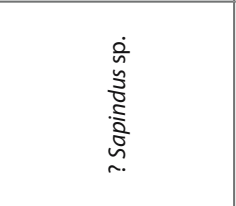 & 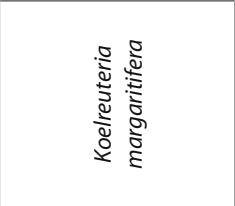 & 1 & 1 & 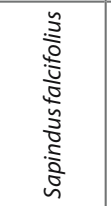 & 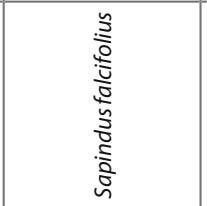 & 1 \\
\hline 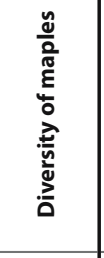 & 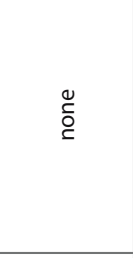 & 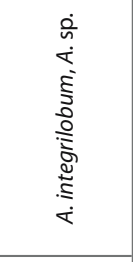 & 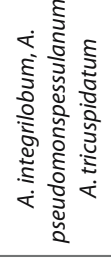 & 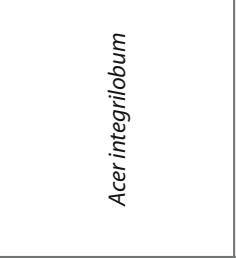 & 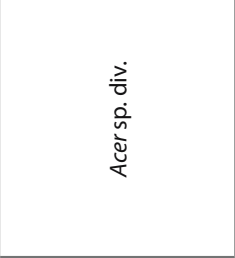 & 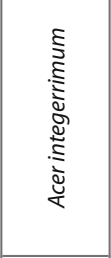 & 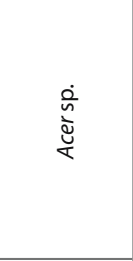 & 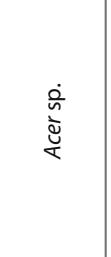 & 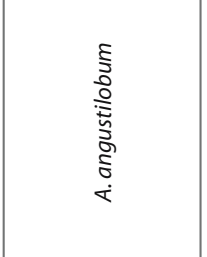 & 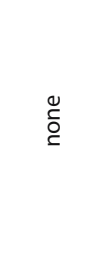 \\
\hline 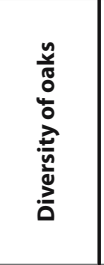 & 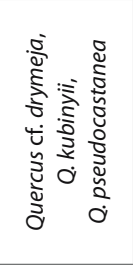 & 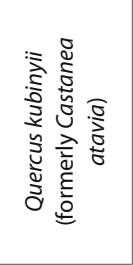 & 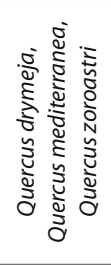 & 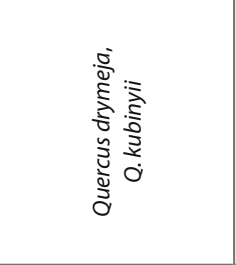 & 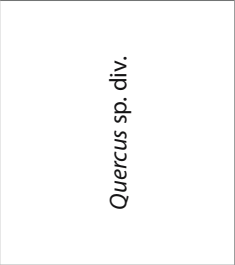 & 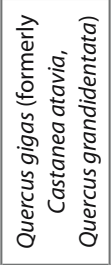 & 1 & 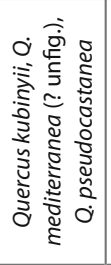 & 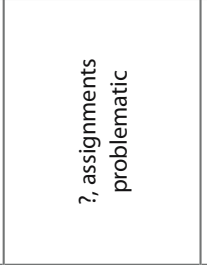 & 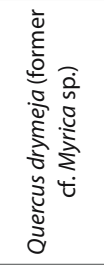 \\
\hline 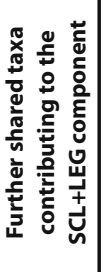 & 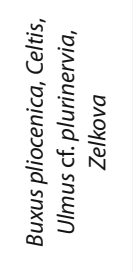 & 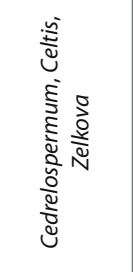 & 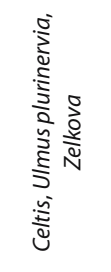 & 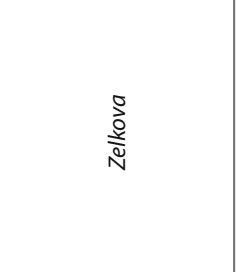 & 咅 & 1 & 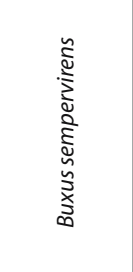 & 1 & 1 & 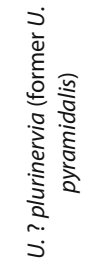 \\
\hline 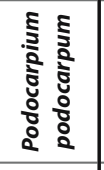 & 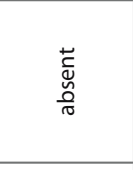 & 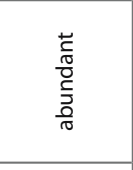 & $\begin{array}{l}\text { 蒿 } \\
\text { 产 }\end{array}$ & 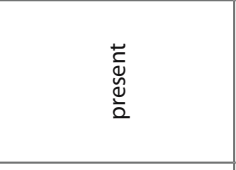 & 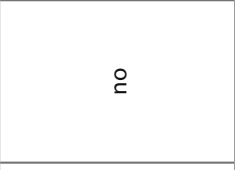 & 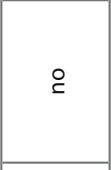 & 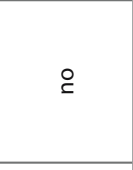 & 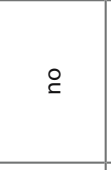 & 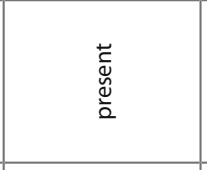 & 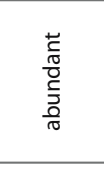 \\
\hline ؛ั & 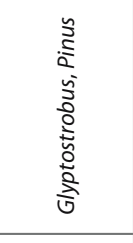 & 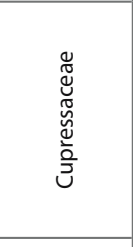 & 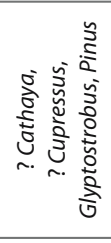 & 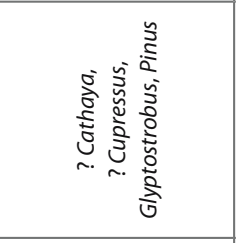 & 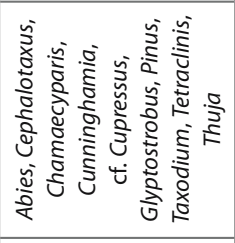 & 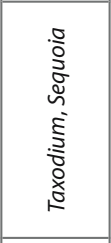 & 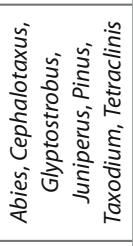 & 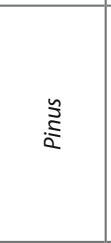 & $\begin{array}{l}\stackrel{0}{\circ} \\
\stackrel{2}{c}\end{array}$ & 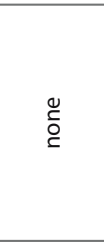 \\
\hline 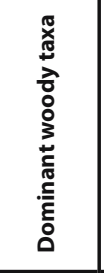 & 1 & 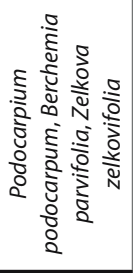 & 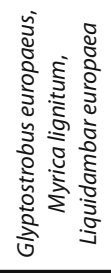 & $\sim$ & n. & n. & r. & $\sim$ & 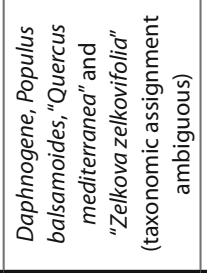 & 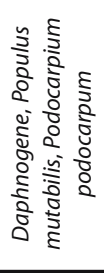 \\
\hline ثี & 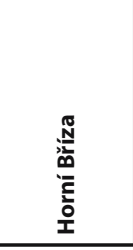 & 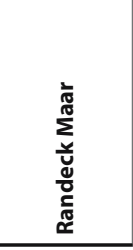 & 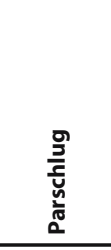 & 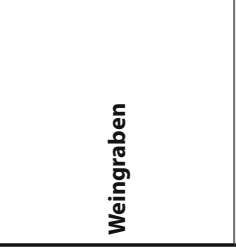 & 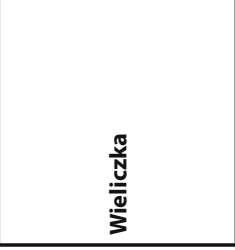 & 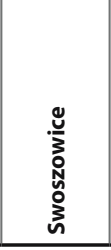 & 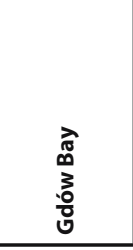 & 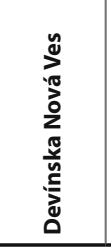 & 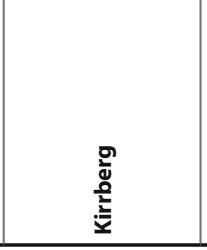 & 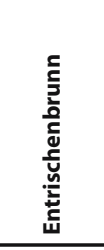 \\
\hline
\end{tabular}




\begin{tabular}{|c|c|c|c|c|c|c|c|c|c|c|}
\hline 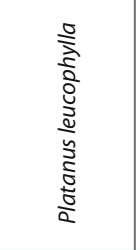 & I & 1 & 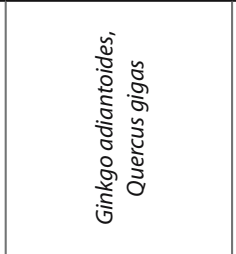 & 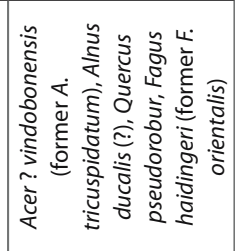 & 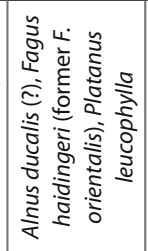 & 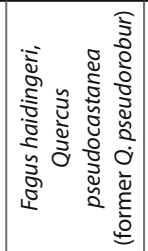 & 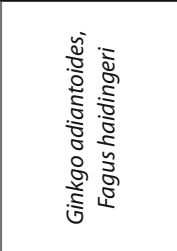 & 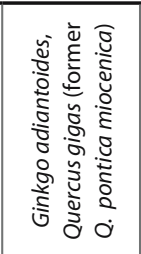 & 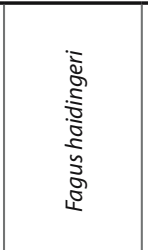 & 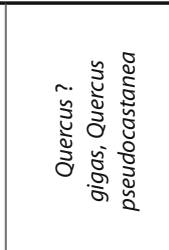 \\
\hline 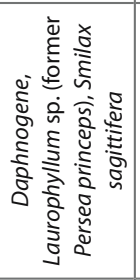 & 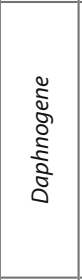 & 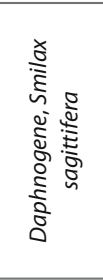 & 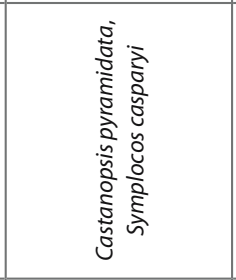 & I & 1 & 1 & 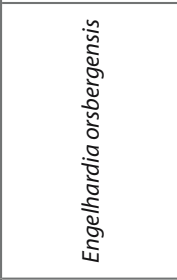 & I & 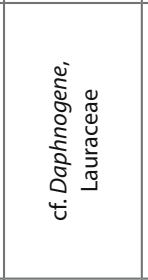 & 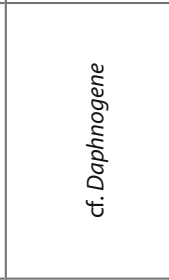 \\
\hline 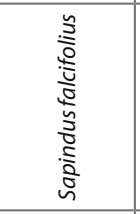 & 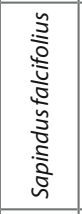 & 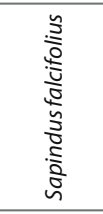 & 1 & 1 & & 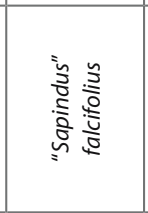 & 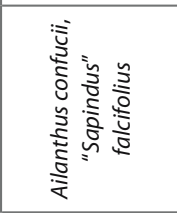 & 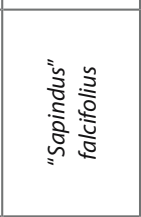 & 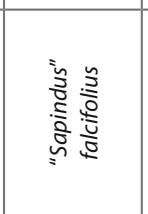 & 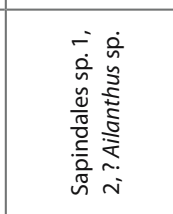 \\
\hline 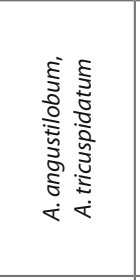 & 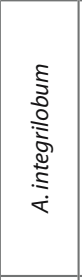 & $\begin{array}{l}0 \\
\stackrel{0}{\check{\sigma}}\end{array}$ & 1 & 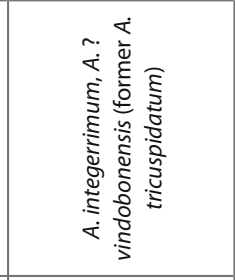 & 总 & 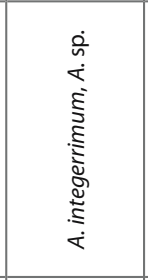 & 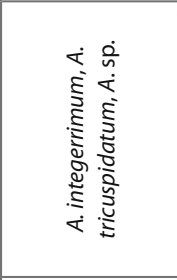 & 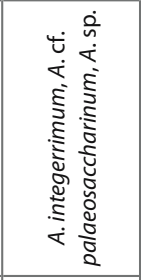 & 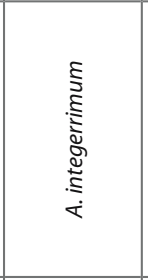 & 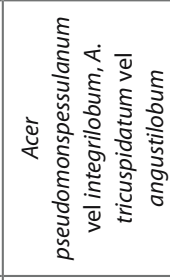 \\
\hline 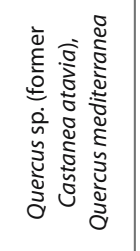 & 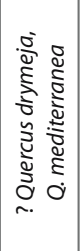 & 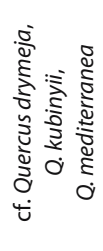 & 1 & 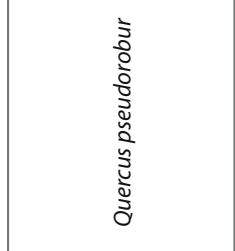 & I & 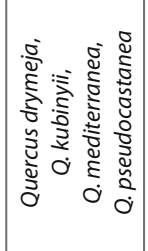 & 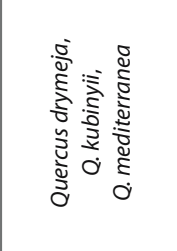 & 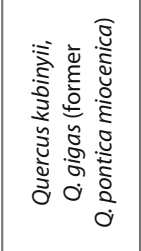 & 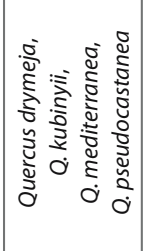 & 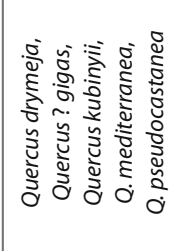 \\
\hline $\begin{array}{l}\text { o } \\
\text { 产 } \\
\text { N }\end{array}$ & 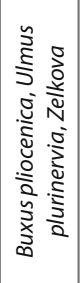 & 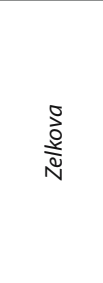 & 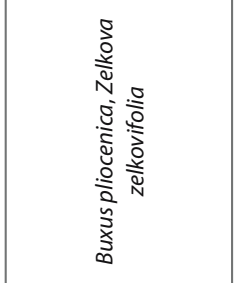 & 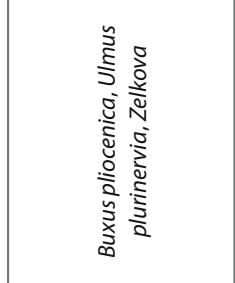 & 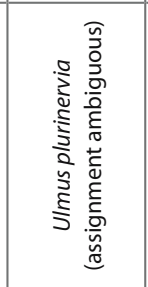 & 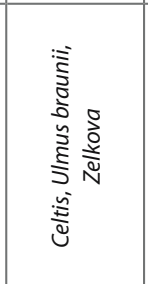 & 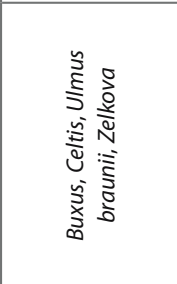 & 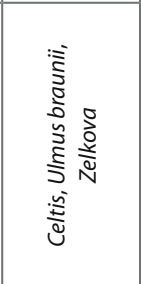 & 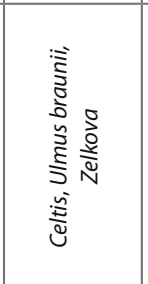 & 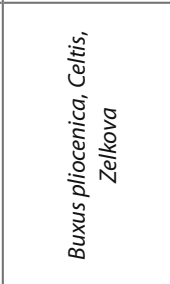 \\
\hline 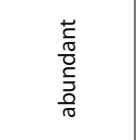 & 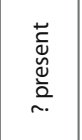 & 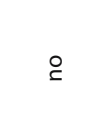 & 1 & 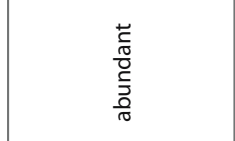 & 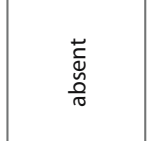 & 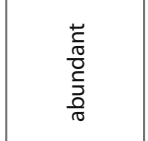 & 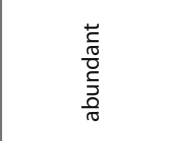 & 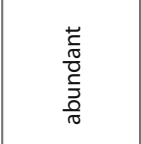 & 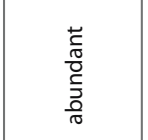 & 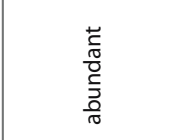 \\
\hline 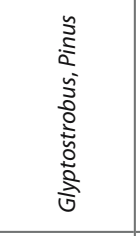 & 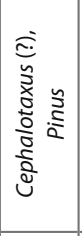 & 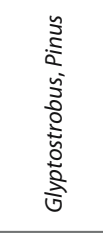 & 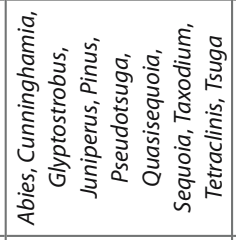 & 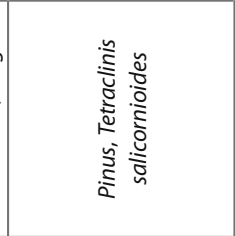 & 峁 & 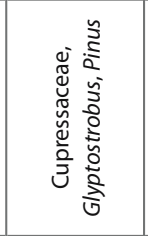 & 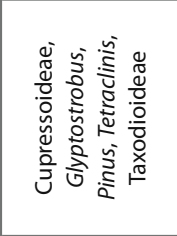 & 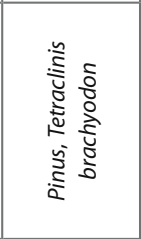 & 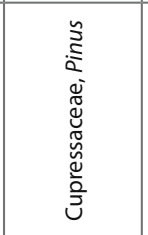 & 吕 \\
\hline 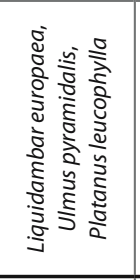 & 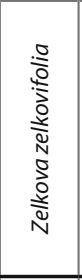 & 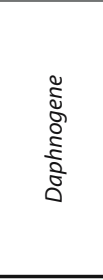 & $\sim$ & 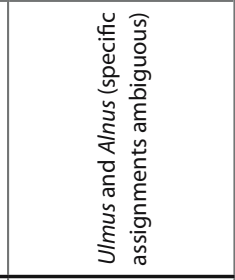 & 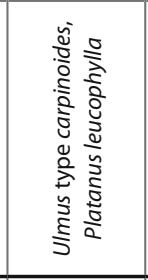 & 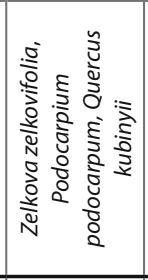 & 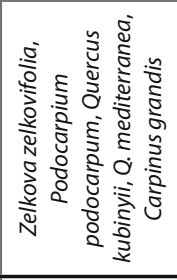 & 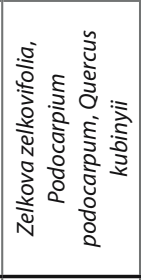 & 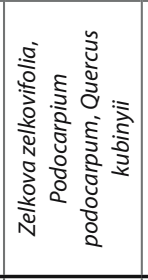 & 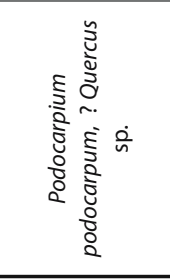 \\
\hline 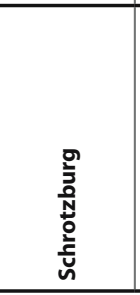 & 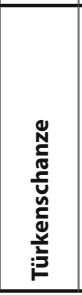 & $\begin{array}{l}\frac{n}{\pi} \\
\stackrel{\tilde{\sigma}}{\bar{\omega}} \\
\\
\end{array}$ & 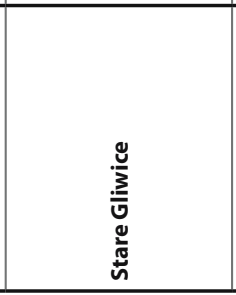 & 商 & 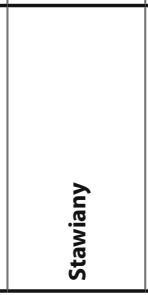 & 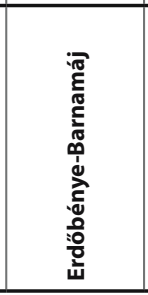 & 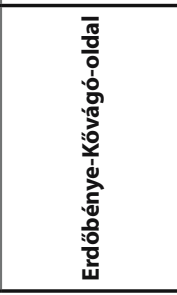 & 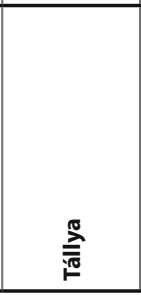 & 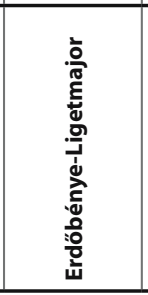 & 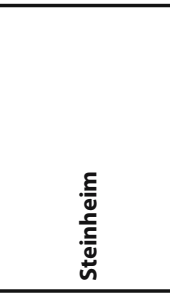 \\
\hline
\end{tabular}




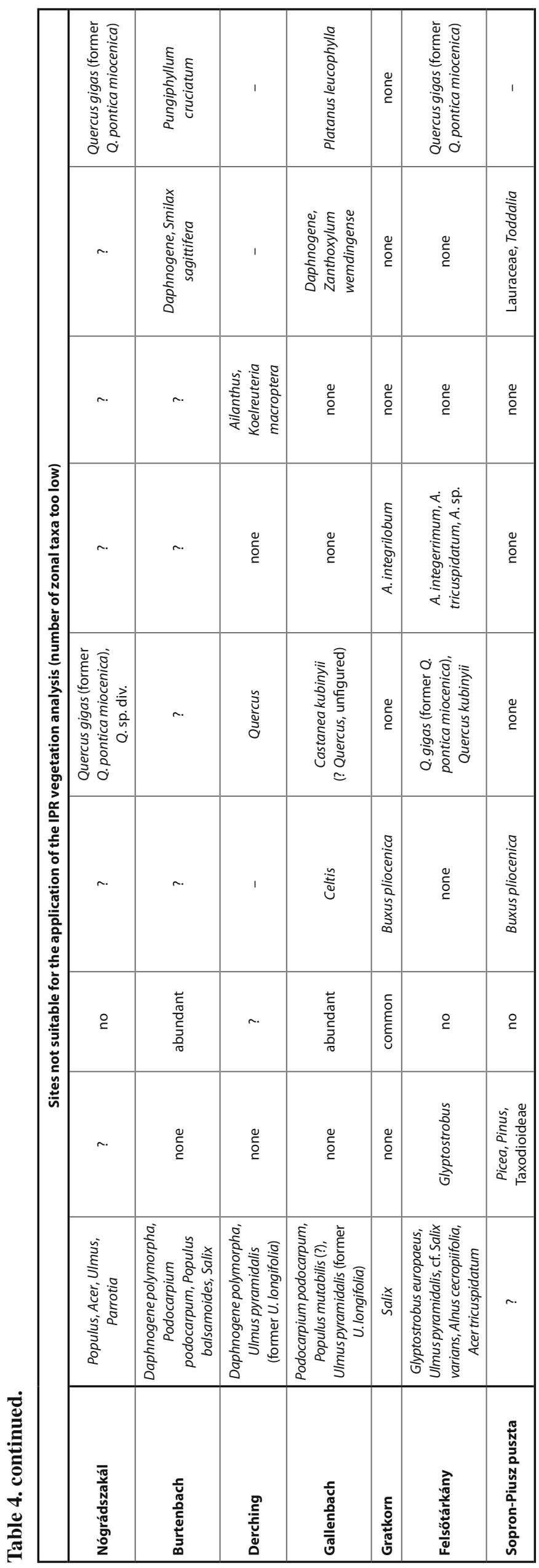

sites the results indicate BLDF (Gdów Bay), ecotone BLDF/ MMF (Swoszowice, Stare Gliwice), MMF (Devínska Nová Ves, Kirrberg) or ecotone MMF/BLEF (Randeck Maarpollen, Weingraben-pollen, Wieliczka). The results for Horní Bříza suggest a transition between MMF/ShSF.

Regarding the floristic composition, the dominant taxa at the different sites are rather variable and no congruent pattern can be identified (Tab. 4). For example, Parschlug is dominated by Glyptostrobus europaeus (Brongniart) Unger, Myrica lignitum (Unger) SAPORTA and Liquidambar europaea A.BRAUN, which is related to the lignite-forming environment there. In Schrotzburg, the most abundant taxa - Liquidambar europaea, Ulmus pyramidalis GöPPERT, Platanus leucophylla (UNGER) KNOBloch, along with Populus balsamoides GöPPERT and P. populina (BRONGNIART) KNOBLOCH - reflect its fluviatile character. The fluviatile influence is also evident at Młyny and Stawiany (Holy Cross Mts.) where Ulmus, Alnus and Platanus leucophylla are most common. In the Paratethys region, at Türkenschanze (Vienna Basin) and the sites in the Tokay Mountains (Erdőbenye-Barnamáj, ErdőbényeKővágó-oldal, Erdőbénye-Ligetmajor, Tállya), Zelkova zelkovifolia (UNGER) BŮžEK et KotLABA is most abundant. In the Tokay Mountains, other common taxa are Podocarpium podocarpum (A.Braun) Herendeen and Quercus kubinyii. In the leaf assemblages, generally, conifers presumably not bound to wetland habitats are largely restricted to pines, except for rare findings of Tetraclinis salicornioides (UNGER) KVAČEK (Weingraben, Wieliczka, Gdów Bay, Stare Gliwice, Erdőbénye Kövágó-oldal, Młyny, Tállya). Sites lacking any records of conifers are Kirrberg, Entrischenbrunn, Stawiany, and Steinheim. Conifer diversity is higher in the pollen and fruit record. At many sites, Podocarpium podocarpum is abundant, at few sites it is at least present (Parschlug, Weingraben, ? Türkenschanze), whereas records are lacking from Horní Bříza, Wieliczka, Swoscowice, Gdów Bay, Devínska Nová Ves, Stare Gliwice, Hernals, Stawiany, Nógrádszakál, Felsőtárkány and Sopron-Piusz puszta.

Furthermore, the following taxa are present at many sites: Buxus pliocenica SAPORTA et Marion, Celtis, Quercus drymeja UNGER, Q. kubinyii, Q. mediterranea Unger, Ulmus type plurinervia UNGER/braunii HEER, and Zelkova zelkovifolia. Oak diversity, including both acutely toothed, partly sclerophyllous forms as well as roburoid ones (type Q. pseudocastanea GöPPERT), is highest in Steinheim (5), Erdőbénye-Barnamáj and Erdőbénye-Ligetmajor (4 each). In the leaf record, entire-margined maples (such as Acer integrilobum WeBER sensu Walther, A. integerrimum Viviani et Massalongo, A. pseudomonspessulanum Unger) occur at more sites (Randeck Maar, Parschlug, Weingraben, Swoszowice, Hernals, Türkenschanze, Młyny, ErdőbényeBarnamáj, Erdőbénye-Kővágó-oldal, Ligetmajor, Tállya, Steinheim, Gratkorn, Felsötárkány) than maples with toothed margins (A. tricuspidatum BRONN and/or A. angustilobum HeER), which are documented from Parschlug, Kirrberg, Schrotzburg, Młyny, Erdőbénye-Kövágó-oldal and Steinheim. No maples have been recorded from Horní Břiza, Entrischenbrunn, Stawiany, Sopron-Piusz puszta, Derching and Gallenbach. This may be an artefact for the latter three sites due to low number of taxa recorded there. Furthermore, many sites share the presence of taxa with compound leaves 
Distance
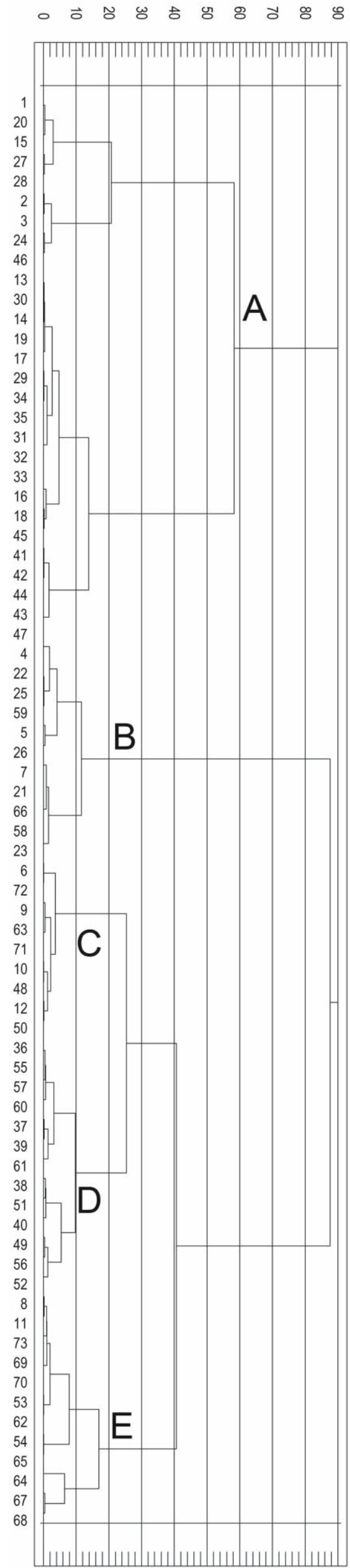

of probable Sapindalean affinity (Ailanthus, Koelreuteria, "Sapindus" and others).

Those sites that did not allow application of the IPR vegetation analysis because they include mainly intrazonal taxa and the number of zonal ones is too low, provide additional signals relevant in this context. Gratkorn (Hably and Meller 2017) includes only 3 taxa (out of 23) that may be regarded primarily as zonal. These are Podocarpium podocarpum, Buxus pliocenica and Acer integrilobum, indicating a close relationship to the plant assemblages richer in zonal taxa. Burtenbach and Gallenbach yield Podocarpium podocarpum; in the latter this legume is abundant. In Felsőtarkany (Erdei and Hír 2002), out of 20 taxa, only Quercus kubinyii, Quercus gigas GöPPERT emend. Walther and Zastawniak (former $Q$. pontica miocenica КUвÁT) and Acer cf. palaeosaccharinum STUR may be regarded as primarily zonal ones.

\section{Results of the cluster analysis}

The comparison of the studied European fossil record with 47 modern vegetation units from temperate to tropical zones of China and Japan (Teodoridis et al. 2011a, 2012) is shown in Text-fig. 3 and Appendix 2. None of the studied fossil sites cluster with subcluster A representing modern BLEF (including MMF of Mt. Emei - 2, 3) and tropical vegetation from SE China and Japan. Swoszowice (58), Gdów Bay (59), and Stare Gliwice (66) belong to subcluster B, representing a close affinity to living BLDF of Mt. Emei (4), Meili Snow Mts. (7), modern Lindera membranaceaFagus crenata (21), and Ilex-Thuja standishii (23) plant communities of BLDF from Shirakami Sanchi and modern Fagus crenata and Vaccinium-Picea regions of Mt. Fuji (25, 26) from Japan. The fossil vegetation units of Tállya (72), Schrotzburg (63) and Erdőbénye Ligetmajor (71) are very close to each other and belong to subcluster $\mathrm{C}$, integrating also modern BLDF (6) and several plant communities of the ShSF dominated by Quercus and Pinus (9), (10), (12) from Meili Snow Mts. (Yunnan, China) sensu Ou et al. (2006). Subcluster D shows close affinity of the fossil sites Randeck Maar (49-52), Devínska Nová Ves (60), Kirrberg (61), Wieliczka (57) and Weingraben-pollen (55) as well as Weingraben-integrated record (56) to modern Eurya-Cryptomeria japonica associations of MMF (36-40) from Yakushima Island (Japan). Subcluster E summarises the fossil assemblages of Steinheim (73), Erdőbénye Barnamáj (69), Erdőbénye Kővágó-oldal (70), Parschlug (53), Entrischenbrunn (62), Weingraben-leaf and -fruit record (54), Hernals (65), Türkenschanze (64), Młyny (67), and Młyny and Stawiany (68) with the modern "Quercus" communities of ShSF from Meili Snow Mts. $(8,11)$.

Text-fig. 3. Results of the cluster analysis based on Ward's method and squared Euclidian distance. Dendrogram showing 5 subclusters (A-E). Cluster analysis is based on the percentages of the BLD, BLE, and SCL+LEG components. Numbers 1-40 sites studied by Teodoridis et al. (2011a), Nos 41-47 by Teodoridis et al. (2012), Nos 48-73 Middle Miocene sites in the wider Central Paratethys region, studied here. The data source is provided in Appendix 2. 


\section{Discussion}

This study is deliberately designed on a rather narrow time interval of about 4 million years with a strong regional focus in order to uncover regional vegetational and climatic peculiarities. The dating of the plant assemblages does not allow for higher resolution in time. No estimations are available regarding how much time is reflected in the individual plant-bearing sediments, nor is an estimation possible as to whether, and to what degree, plant taphocoenoses accumulated with an overlap in time.

The strong signal towards ShSF derives from the sites in the Pannonian Basin, the North Alpine and Carpathian Foredeeps, the adjacent Parschlug Basin, and the Swabian Alb. Ecotone between MMF/BLEF, indicated by Wieliczka, Randeck Maar-pollen, and Weingraben-pollen, contrasts with the leaf and fruit record from Randeck Maar and Weingraben, which signals ShSF. Such differences in the pollen versus the leaf and fruit signal are probably related to the lower taxonomic resolution of the pollen record and to the fact that pollen assemblages may be much more strongly influenced by long-distance transport than the macro record (Kovar-Eder and Kvaček 2007). Note for Wieliczka, Randeck Maar-pollen and Weingraben-pollen that the results (ecotone $\mathrm{MMF} / \mathrm{BLEF}$ ) differ from the formerly presented ones (BLEF; Kovar-Eder and Kvaček 2007, Kovar-Eder et al. 2008). This is due to the fine-tuning of the IPR vegetation analysis, i.e. raising the threshold of the BLE component from $30 \%$ to $40 \%$ for BLEF (Teodoridis et al. 2011a). Dated by the flora, the age of the assemblage from Horní Bříza, which points to the ecotone $\mathrm{MMF} / \mathrm{ShSF}$, is less confident. No further interpretation of this result should be advanced at this time.

In the Carpathian Foredeep, the differences in vegetation proxies from the Badenian sites (Wieliczka ecotone MMF/ BLEF, Swoszowice and Stare Gliwice ecotone BLDF/MMF, Gdów Bay BLDF) compared to the Early Sarmatian ones (Młyny, Młyny and Stawiany both ShSF) may be related to differences in age. Our results for Stare Gliwice are somewhat contradictory to Szafer's (1961), who recognised a distinct "Mediterranean" element there (e.g. Juniperus, Buxus, Cotinus). These taxa are, however, by far outnumbered by broad-leaved deciduous ones, explaining the results of the IPR vegetation analysis. The signal from the Carpathian Foredeep (BLDF to ecotone MMF/BLEF) compared to the other regions at that time may reflect regional vegetation differentiation due to either more humid and/or a more equal year-round distribution of precipitation there.

In the North Alpine Foredeep, Podocarpium podocarpum is a characteristic element of the floras assigned to phytozone OSM 3b sensu Gregor (1982). The understanding of $P$. podocarpium as a wetland-species and indicative of humid climatic conditions assumed that it is closely related to Gleditsia aquatia Marshall and G. heterophylla Bunge (Gregor and Hantke 1980). Herendeen (1992) has, however, demonstrated that $P$. podocarpum has no contemporary equivalent. In the fossil record, $P$. podocarpum occurs not only in plant assemblages of strong intrazonal character such as the sites in the North Alpine Foredeep, but is also abundant at sites that mainly reflect zonal environments such as Randeck Maar, the Erdőbénye sites, and Steinheim.
This explains our interpretation of it being a mainly zonal taxon with a wide ecological range.

The here-presented results in which ShSF is the dominant zonal vegetation unit in this region contrasts with those obtained for preceding and later Miocene periods (Kovar-Eder and Kvaček 2007, Kovar-Eder et al. 2008, and see below). ShSF as an important zonal vegetation unit in the Paratethys region during the Middle Miocene was not clearly recognised by Kovar-Eder et al. (2008) because the time interval 17-14 m.a. (late Early Miocene to early Middle Miocene) was not suitable and, covering the entire area of Western Eurasia meant that study was on too large a scale.

\section{Vegetation characteristics during the Middle Miocene}

Due to the ongoing discussions regarding the vegetation and climate in the Langhian/Serravallian (e.g. Hably and Meller 2017), we here attempt to outline a feasible picture of the landscape and vegetation. In the Langhian/ Serravallian time interval, the Central Paratethys was highly dissected by islands and the arising Carpathians (Text-fig. $1)$; tectonic activity was connected to volcanism. The fossil record is rich, i.e. from former islands in the Pannonian Basin (Tokay Mountain sites related to volcanism), the Carpathian Foredeep (Wieliczka, Swoszowice, Gdów Bay, Stare Gliwice, Młyny, Stawiany), from western parts of the Pannonian Basin system (Weingraben, Devínska Nová Ves, Sopron-Piusz puszta, Türkenschanze, Hernals), from floodplain environments at the NW margin of the Styrian Basin (Gratkorn), and the Parschlug lignite basin in the Norian depression. Along the North Alpine Foredeep, in its western part, fluviatile-lacustrine domains prevailed (e.g. Schrotzburg, Entrischenbrunn, Kirrberg). On the Swabian Alb, which was a karst plateau at that time, maar lake deposits (Randeck Maar) and the deposits of an impact crater lake (Steinheim) record the surrounding vegetation. Finally, the flora from Horní Břiza represents the record from fluviolacustrine sediments of the Plzen Basin in the southern part of the Bohemian Massif (Text-fig. 1, Tab. 1).

Intrazonal vegetation developed along streams, braided rivers, floodplains, and lakes. Characteristic are, among others, Acer tricuspidatum, A. angustilobum, Juglandaceae, Liquidambar europaea, Platanus leucophylla, Populus, Salix and Ulmus pyramidalis. In particular braided river systems and floodplains probably occupied vast regions with very diverse habitats ranging from almost mesophytic to riparian and floodplain forests, to swampy and aquatic habitats. These environments, which are best represented in the fossil record, were most dynamic due to major factors such as water discharge, tectonics and sea level changes.

Including the pollen record, Kvaček et al. (2006) were able to delineate the altitudinal vegetation zonation. Conifers (e.g. Abies, Keteleeria, Pseudotsuga) and mainly broadleaved deciduous woody taxa formed extrazonal forests at higher altitudes. Such vegetation is reflected in the pollen record of Randeck Maar, Weingraben and Devínska Nová Ves. ShSF developed as zonal vegetation in mesophytic colline and lowland environments. Northwards, zonal vegetation turns into the ecotones between MMF/BLDF and MMF/BLEF. ShSFs were characterised by broadleaved deciduous trees and shrubs (BLD component mainly 
around 45-60\%), trees and shrubs with sclerophyllous and legume-like foliage (SCL+LEG component around 20$40 \%$ ) and broad-leaved evergreen ones (BLE component around 5-29\%). They differ in composition from that of earlier and later periods in several aspects. Oak diversity is high, including a mixture of both broad-leaved deciduous (Quercus gigas, roburoid oaks type $Q$. pseudocastanea) and more or less sclerophyllous, probably also deciduous ones ( $Q$. mediterranea, $Q$. drymeja, $Q$. kubinyii), including both more ancient ( $Q$. drymeja) and younger ( $Q$. gigas, Q. pseudocastanea) species. Podocarpium podocarpum is among the most common species, often occurring in great quantities (e.g. Randeck Maar, Erdőbénye sites, sites along the North Alpine Foredeep, Steinheim). For Hungary, Hably (1992) lists the following additional occurrences: Gyöngyöspata, Szurdokpüspöki and Sajómercse (all Badenian) and Bánhorváti, Bújak, Diósgyoőr and Füzérradvány (all Sarmatian in age). Further taxa occurring at numerous sites and often abundant are elms with smallsized foliage of the type Ulmus plurinervia/U. braunii and Zelkova zelkovifolia. Buxus pliocenica, Celtis and entiremargined maples (Acer integerrimum, A. integrilobum, A. pseudomonspessulanum) occur commonly but usually are not abundant. Taxa with compound leaves of Sapindalean affinity (such as Rutaceae, Simaroubaceae, Sapindaceae) but of yet often uncertain generic assignment occur at many sites; their leaflets are sometimes abundant (e.g. Steinheim, Randeck Maar). Conifers are both scarce and low in diversity, except for pines. A remarkable feature of the Middle Miocene plant record is the first occurrence of broad-leaved deciduous taxa such as Acer vindobonensis (EtTingshausen) Berger, Ginkgo adiantoides (UNGER) Heer, Platanus leucophylla and Quercus pseudocastanea (Kovar-Eder et al. 1994). Some ancient taxa such as Engelhardia orsbergensis (WEBER) JÄHNICHEN, MAI et WALTHER/macroptera (BRONGNIART) UNGER, Lauraceae (especially Daphnogene), and Tetraclinis salicornioides may also occur. Records of palms are extremely rare, restricted to the oldest sites Horní Brríza and Randeck Maar and to Stare Gliwice. The zonal vegetation was diversified depending on the soil type, sun exposure, and altitude. Erdei et al. (2007) recognised this unique floristic character in the Hungarian Sarmatian, stressing also its "subxerophytic" aspect and suspecting that this may be related to the volcanic environments. We demonstrate here that this vegetation type had a wider spatio-temporal distribution, although it appears restricted to the (latest Early) and Middle Miocene. KovarEder et al. (2012) demonstrated that the vegetation during the late Early and early Middle Miocene was characterised by an increased proportion of anemochorous woody taxa and, vice versa, decreasing endozoochorous taxa, contrary to that of preceding periods. These shifts in dispersal syndromes indicate changes in diet sources for herbivores. We suspect that these trends would appear even more pronounced if the Early and Middle Miocene records were separated properly, as done here.

Comparing the fossil record to modern vegetation in China and Japan by applying the cluster analysis and/or IPR vegetation analysis, the Middle Miocene plant record shows a close affinity to ShSF vegetation (Meili Snow Mt., Yunnan, China), which is distributed at 2,580-3,650 m alt.
This vegetation type is characterised by sclerophyllous oaks, i.e. Quercus aquifolioides REHDER et E.H.WILsON, Q. gilliana REHDER et E.H.WILSON, Q. guyavifolia REHDER et E.H.WILSON and $Q$. rehderiana HANDEL-MazzetTI in association with Rhododendron, Populus, Myrsine, Lonicera, Dipelta, Rosa, Salix, Urtica, Rubus, Picea, Larix and Drynaria. Such forests are relatively frequent in northwest Yunnan and western Sichuan and are comparable to sclerophyllous forests in the Mediterranean region (Wu et al. 1987, Ou et al. 2006) and in California, USA (Tang 2006).

The here-presented results support the view expressed by several authors (e.g. Jing 1981, Zhou et al. 2003, Tang 2006) that modern evergreen sclerophyllous oak forests in China presumably evolved from sclerophyllous Quercus forests in the former Tethys region after the uplift of the Himalayas.

\section{The Middle Miocene plant record compared to the Early and Late Miocene}

Separating the Early Miocene from the Middle Miocene plant assemblages (compare Kovar-Eder et al. 2008: appendix 6) reveals that the former usually differ by having higher proportions of the BLE component and lower ones of the SCL+LEG component. This explains BLEF or MMF, respectively, as the major vegetation units. The flora from Randeck Maar, which is correlated to MN 5, indicates that ShSF probably started to spread in the outgoing Early Miocene/earliest Middle Miocene. We assume that the time frame 17-14 m.a. set for the afore-mentioned study was not suitable to detect these changes in the dominating vegetation unit from BLEF/MMF in the Early Miocene to ShSF in the here-studied region in the Middle Miocene.

In both Early and Middle Miocene plant assemblages, the BLD component is similarly low. The first occurrences of several broad-leaved deciduous taxa such as Acer vindobonensis, Alnus ducalis GAUDIN emend. Knobloch, Fagus, Ginkgo adiantoides, Platanus leucophylla, and Quercus pseudocastanea (Kovar-Eder et al. 1994) do, however, indicate dynamic changes in the composition of the BLD component during the Middle Miocene. In the Late Miocene the BLD component is usually distinctly higher, often exceeding $80 \%$. The LEG+SCL component is similarly low in the Early Miocene and Tortonian but distinctly higher in the Langhian/Serravallian. Due to the diversity of broad-leaved evergreen taxa of Fagaceae, Lauraceae, Magnoliaceae, Theaceae, and Symplocaceae, the BLE component is usually highest in plant assemblages from the Early Miocene. In contrast, its values are similarly low in the Langhian/Serravallian and Tortonian. Our interpretation is that the increase of sclerophyllous and legume-type taxa occurred at the cost of broad-leaved evergreen ones from the late Early Miocene onwards. The increase of broadleaved deciduous taxa occurred in the Tortonian more at the cost of sclerophyllous and legume-type taxa than of broadleaved evergreen ones. Podocarpium podocarpum already appeared in the Early Miocene, e.g. Čermníky (Bůžek 1971), and developed a bloom in the Langhian/Serravallian. It became extinct during the Early Pannonian (Hably 1992). In the Tortonian, the diversity of deciduous broad-leaved Betulaceae, Fagaceae, Hamamelidaceae, Juglandaceae, Sapindaceae (Acer), and others accounts for the overall 
higher values of the BLD component (Kovar-Eder et al. 2008). In Late Miocene taphocoenoses, the proportion of the BLD component is usually higher than that in the Early and Middle Miocene assemblages. In contrast, the SCL+LEG component is distinctly lower than in the plant assemblages from the Langhian/Serravallian: its values are more similar to those retrieved from most Early Miocene sites.

\section{Comparison with existing vegetation reconstructions}

Kvaček et al. (2006) presented palinspastic maps of the Miocene evolution of landscape and vegetation. These maps clearly indicate "sub-humid aspects" in southern parts of the Paratethys (Hungary, Serbia, Romania) and an increase in broad-leaved deciduous elements in more northern regions of the Paratethys during the Late Badenian. Utescher et al.'s (2007) assessment of tree diversity and vegetation, scoring the Miocene plant record to functional types (PFT), is more difficult to compare. This is because the PFTs are not congruent with the components defined for the IPR vegetation analysis. Moreover, the plant record is treated in an undifferentiated manner to assess the overall vegetation, contrary to the IPR vegetation analysis, which differentiates plants according to the presumable autecology of the taxa. We assume that "PFT 9 broadleaved evergreen - warm temperate/dry season" is similar to the SCL component in the IPR vegetation analysis. Cluster analysis of the PFT scores groups Öhningen (comparable to Schrotzburg), Randeck Maar, Türkenschanze, Erdőbénye-Ligetmajor and Młyny in cluster (M2). In this cluster, PFT 9 is of significance, indicating elevated values of woody plants adapted to seasonal drought, similar to the results of the IPR vegetation analysis. Otherwise, the PFT approach delivers distinctly higher proportions of broad-leaved deciduous taxa than obtained by the IPR vegetation analysis. This most likely reflects the undifferentiated treatment of the zonal and intrazonal taxa by the PFT analysis. Accordingly, the vegetation assessment based on the PFT approach is considered less convincing.

In the context of the modern vegetation units of China and Japan, as applied here, the Tortonian plant assemblages from Crete (Gavdos, Makrilia, Pitsidia) also plot with ShSF (Mantzouka et al. 2015) as does most of the Langhian/ Serravallian record from the wider Central Paratethys region. Floristically, especially Makrilia and Pitsidia show distinct similarities to our Central European record as reflected by the diversity of oaks and the occurrence of Buxus pliocaenica, Podocarpium podocarpum and others (Sachse et al. 1999, Zidianakis et al. 2010). This highlights the evolutionary linkage between ShSF vegetation in both regions.

\section{Vegetation evolution signals climate change}

Although the IPR vegetation analysis is not designed for direct climate assessment, the resulting vegetation development not only reflects regional landscape development and related vegetation change, but also points to overall climate change in this region.

Today, ShSFs are bound to climates with pronounced seasonal changes in precipitation. Based on the here-studied record, the Langhian/Serravallian period experienced more marked precipitation seasonality than the preceding and following periods. The onset of such climatic conditions occurred around the turn of the Early to Middle Miocene, documented by the fossil floras from Randeck Maar (MN 5). The Swabian Alb may have been suitable for the early appearance of ShSF due to the hydrological regime characterising karst plateaus.

The development of the BLD component (similarly low values in the Early and Middle Miocene, distinctly higher ones in the Tortonian) points to a stronger temperature drop around the turn of the Middle/Late Miocene than in the preceding Miocene time. This supports Böhme's (2003) assumption that the changes in the precipitation regime (towards a seasonal dry climate) in the Early Badenian were probably less linked to changes in the temperature regime. The first records of several broad-leaved deciduous woody plants (see above) in the wider Central Paratethys region cannot be further resolved stratigraphically to underpin a drastic drop in mean annual temperature around the Langhian/Serravallian turn as assumed by Böhme (2003).

Based on the herpetofauna, Böhme (2003) and Böhme et al. $(2008,2011)$ suggest strong seasonality in precipitation in the Early Langhian, dry climatic conditions in the later Langhian, and dry conditions during the interval Late Serravallian/Early Tortonian. For the wider Central Paratethys region, the plant record indicates seasonal drought, but the stratigraphic resolution is insufficient to recognise possible oscillations.

Based on the Coexistence Approach (CA) (Mosbrugger and Utescher 1997), the record of fossil wood from the North Alpine Foredeep in southern Germany served for another attempt to reconstruct the vegetation and climate (Böhme et al. 2007). Those results indicate an almost equal distribution of precipitation year-round and, overall, humid conditions from the Late Ottnangian through the Middle Badenian. Our results, which include several plant assemblages from the North Alpine Foredeep, do not confirm such climatic conditions for the Badenian.

Bruch et al. (2011) presented precipitation estimates for Central Europe throughout the Miocene based on the evaluation of 169 plant-bearing sites by the CA. Several of the sites they evaluated palaeoclimatically are also included in our study. Those authors, however, arrive at very high humidity during the Langhian, followed by slightly lower humidity and seasonality in the Serravallian and slightly increased precipitation and seasonal changes during the Tortonian. Their results for the Langhian in particular are not in agreement with ours nor with those derived from the herpetofauna (Böhme et al. 2011). Otherwise, their compilation is too rough for deciphering variability in humidity at smaller regional and shorter time scales. We assume that the CA proxies reveal high humidity values and minor variability because this method neglects autecological differentiation. Furthermore, it relies on so-called "nearest living relatives", which more precisely should be termed "most similar living relatives" because though most similar, they may not be the closest relatives. Taxa of unknown or problematic taxonomic affinity must be excluded from the CA. Such taxa are, however, not uncommon in the Miocene (e.g. Cedrelospermum, "Sapindus" falcifolius (A.BRAUN) A.BrAUN), and especially small-sized, sclerophyllous leaves are often of unknown taxonomic affinity. In contrast, the IPR 
vegetation analysis includes such taxa to the extent possible, evaluating leaf physiognomy.

\section{Conclusions}

The Middle Miocene plant record in the wider Central Paratethys region provides a consistent signal pointing to vegetation adapted to seasonal changes in precipitation. This signal was already recognised by many authors for the Early Sarmatian (e.g. Berger and Zabusch 1953, Andreánszky 1959, Szafer 1961, Gregor 1982) and, subsequently, was interpreted very controversially both in terms of vegetation and climate. Our study of mainly independently dated fossil floras with the spatio-temporal focus on the Langhian/ Serravallian of the Central Paratethys region reveals that ShSF was a wide-spread vegetation unit at low altitudes during that period (i.e. zonal vegetation). Its spread may have already started there during the latest Early Miocene. Constraints on stratigraphic correlation hinder, however, tracing possible oscillations in vegetation and climate.

Forest dynamics, namely floristic composition, must be envisioned as depending mainly on soil type and sun exposure, but also on altitude. Broad-leaved taxa preferred more humid sites (e.g. north-exposed slopes) than sclerophyllous and legume-type taxa. The Langhian/ Serravallian record of likely zonal taxa differs both floristically and leaf-physiognomically from that of both preceding and later Miocene periods. This becomes evident only when the fossil record, which is usually strongly dominated by intrazonal taxa, is differentiated according to taxa preferring zonal or intrazonal habitats. Intrazonal environments (along river systems, floodplains, swamps, lake margins, etc.) and their specific plant communities occupied vast regions, e.g. in the North Alpine Foredeep. Extrazonal vegetation (vegetation at higher altitudes) is mainly reflected in the pollen record.

The zonal vegetation of the Langhian/Serravallian is comparable to that occurring today under natural conditions in the northern hemisphere with a climate at the transition between $\mathrm{Cs}$ to $\mathrm{Cfa} / \mathrm{Cfb}$ (sensu Köppen).

\section{Acknowledgements}

We would like to thank Lilla Hably, Mathias Harzhauser and Reinhard Sachsenhofer for information regarding age constraints of single plant sites. Greatly appreciated are also the suggestions and notes made on the submitted version of the manuscript by the reviewers Z. Kvaček and T. Utescher. This research was supported by the grants GAČR 18-25067S and Progres Q17 of the Charles University.

\section{References}

Andreánszky, A. (1959): Die Flora der Sarmatischen Stufe in Ungarn. - Akadémia Kiadó, Budapest, 360 pp.

Bachmayer, F., Rögl, F., Seemann, R. (1991): Geologie und Sedimentologie der Fundstelle miozäner Insekten in Weingraben (Burgenland, Österreich). - In: Lobitzer, H., Császár, G. (eds), Jubiläumsschrift 20 Jahre Geologische
Zusammenarbeit Österreich-Ungarn, Teil 1. Geologische Bundesanstalt, Wien, pp. 53-70.

Berger, J.-P., Reichenbacher, B., Becker, D., Grimm, M., Grimm, K., Picot, L., Storni, A., Pirkenseer, C., Derer, C., Schaefer, A. (2005): Paleogeography of the Upper Rhine Graben (URG) and the Swiss Molasse Basin from Eocene to Pliocene. - International Journal of Earth Sciences, 94: 6947-710.

https://doi.org/10.1007/s00531-005-0475-2

Berger, W. (1951): Pflanzenreste aus dem tortonischen Tegel von Theben-Neudorf bei Pressburg. - Sitzungsberichte der Österreichischen Akademie der Wissenschaften, mathematisch-naturwissenschaftliche Klasse, Abt. 1, 160: 273-278.

Berger, W. (1953): Pflanzenreste aus den obermiozänen Ablagerungen von Wien-Hernals. - Annalen des Naturhistorischen Museums Wien, 59: 141-154.

Berger, W., Zabusch, F. (1953): Die obermiozäne (sarmatische) Flora der Türkenschanze in Wien. - Neues Jahrbuch für Geologie und Paläontologie, Abhandlungen, 98(2): 226-276.

Böhme, M. (2003): The Miocene Climatic Optimum: evidence from ectothermic vertebrates of Central Europe. Palaeogeography, Palaeoclimatology, Palaeoecology, 195(3-4): 389-401. https://doi.org/10.1016/S0031-0182(03)00367-5

Böhme, M., Bruch, A. A., Selmeier, A. (2007): The reconstruction of Early and Middle Miocene climate and vegetation in Southern Germany as determined from the fossil wood flora. - Palaeogeography, Palaeoclimatology, Palaeoecology, 253: 91-114. https://doi.org/10.1016/j.palaeo.2007.03.035

Böhme, M., Ilg, A., Winklhofer, M. (2008): Late Miocene "washhouse" climate in Europe. - Earth and Planetary Science Letters, 275(3-4): 393-401. https://doi.org/10.1016/j.eps1.2008.09.011

Böhme, M., Winklhofer, M., Ilg, A. (2011): Miocene precipitation in Europe: Temporal trends and spatial gradients. - Palaeogeography, Palaeoclimatology, Palaeoecology, 304: 212-218. https://doi.org/10.1016/j.palaeo.2010.09.028

Bruch, A. A., Utescher, T., Mosbrugger, V. (2011): Precipitation patterns in the Miocene of Central Europe and the development of continentality. - Palaeogeography, Palaeoclimatology, Palaeoecology, 304: 202-211. https://doi.org/10.1016/j.palaeo.2010.10.002

Bůžek, Č. (1971): Tertiary Flora from the Northern Pětipsy Area (North-Bohemian Basin). - Rozpravy Ústředního ústavu geologického, 36: 1-118.

Cohen, K. M., Finney, S. C., Gibbard, P. L., Fan, J.-X. (2013; updated): The ICS International Chronostratigraphic Chart. - Episodes, 36: 199-204.

Doppler, G., Heissig, K., Reichenbacher, B. (2005): Die Gliederung des Tertiärs im süddeutschen Molassebecken. - Newsletter on Stratigraphy, 41: 359-375. https://doi.org/10.1127/0078-0421/2005/0041-0359

Draxler, I., Zetter, R. (1991): Palynologische Untersuchungen in den mittel-miozänen Hochriegelschichten (Süßwasserschichten) von Weingraben (Gemeinde Kaisersdorf, Burgenland, Österreich). - In: Lobitzer, H., 
Császár, G. (eds), Jubiläumsschrift 20 Jahre Geologische Zusammenarbeit Österreich-Ungarn, Teil 1. Geologische Bundesanstalt, Wien, pp. 71-92.

Erdei, B., Hably, L., Kázmér, M., Utescher, T., Bruch, A. A. (2007): Neogene flora and vegetation development of the Pannonian domain in relation to palaeoclimate and palaeogeography. - Palaeogeography, Palaeoclimatology, Palaeoecology, 253: 115-140.

https://doi.org/10.1016/j.palaeo.2007.03.036

Erdei, B., Hír, J. (2002): Vegetation and climate reconstruction of Sarmatian (Middle Miocene) sites from NE and W Hungary. - Acta Universitatis Carolinae, Geologica, 46(4): 75-84.

Erdei, B., Lesiak, M. (1999-2000): A study of dispersed cuticles fossil seeds and cones from Sarmatian (Upper Miocene) deposits of Sopron-Piusz puszta (W Hungary). - Studia Botanica, 30-31: 5-26.

Ettingshausen, C. von (1851): Die Tertiär-Floren der Österreichischen Monarchie. No. 1. Fossile Flora von Wien.Abhandlungen der kaiserlich-königlichen Geologischen Reichsanstalt, 2: 1-36.

Ettingshausen, C. von (1878): Beiträge zur Kenntniss der fossilen Flora von Parschlug in Steiermark. - Denkschriften der kaiserlichen Akademie der Wissenschaften, mathematisch-naturwissenschaftliche Classe, 38: 81-92.

Gregor, H.-J. (1980): Ein neues Klima- und Vegetations-Modell für das untere Sarmat (Mittelmiozän) Mitteleuropas unter spezieller Berücksichtigung floristischer Gegebenheiten. - Verhandlungen der Geologischen Bundesanstalt, 1979(3): 337-353.

https://doi.org/10.1002/fedr.19800910303

Gregor, H.-J. (1982): Die jungtertiäre Floren Süddeutschlands - Paläokarpologie, Phytostratigraphie, Paläoökologie, Paläoklimatologie. - F. Enke Verlag, Stuttgart, $278 \mathrm{pp}$.

Gregor, H.-J., Hantke, R. (1980): Revision der fossilen Leguminosengattung Podogonium (HeER) (=Gleditsia LiNNÉ) aus dem europäischen Jungtertiär. - Feddes Repertorium, 91(3): 151-182.

Gross, M., Böhme, M., Havlik, P., Aigistorfer, M. (2014): The Late Middle Miocene (Sarmatian s.str.) fossil site Gratkorn - the first decade of research, geology, stratigraphy and vertebrate fauna. - Palaeobiodiversity and Palaeoenvironments, 94: 5-20. https://doi.org/10.1007/s12549-013-0149-1

Hably, L. (1992): Distribution of legumes in the Tertiary of Hungary. - In: Herendeen, P. S., Dilcher, D. L. (eds), Advances in Legume Systematics, Part 4. The Fossil Record. Royal Botanic Gardens, Kew, pp. 169-187.

Hably, L., Meller, B. (2017): Sarmatian wetlands at the NW of the Pannonian Basin system (Gratkorn, Styrian Basin, Austria, late middle Miocene). Part 1. The leaf record. Palaeontographica, Abt. B, 295: 135-165. https://doi.org/10.1127/palb/295/2017/135

Hantke, R. (1954): Die fossile Flora der obermiozänen Oehninger-Fundstelle Schrotzburg (Schienerberg, Süd-Baden). - Denkschriften der Schweizerischen Naturforschenden Gesellschaft, 80(2): 1-118.

Harzhauser, M., Gross, M., Binder, H. (2008): Biostratigraphy of Middle Miocene (Sarmatian) wetland systems in an Eastern Alpine intramontane basin (Gratkorn Basin,
Austria): the terrestrial gastropod approach. - Geologica Carpathica, 59(1): 45-58.

Harzhauser, M., Piller, W. E. (2007): Benchmark data of a changing sea - Palaeogeography, palaeobiogeography and events in the Central Paratethys during the Miocene. - Palaeogeography, Palaeoclimatology, Palaeoecology, 253: 8-31. https://doi.org/10.1016/j.palaeo.2007.03.031

Heer, O. (1855-1859): Flora tertiaria Helvetiae I-III. - Verlag der Lithographischen Anstalt von J. Wurster \& Compagnie, Winterthur, $117+110+378 \mathrm{pp}$.

Heizmann, E. P. J., Reiff, W. (2002): Der Steinheimer Meteorkrater. - Verlag Dr. Friedrich Pfeil, München, 160 pp.

Henrot, A.-J., Utescher, T., Erdei, B., Dury, M., Hamon, N., Ramstein, G., Krapp, M., Herold, N., Goldner, A., Favre, E., Munhoven, G., François, L. (2017): Middle Miocene climate and vegetation models and their validation with proxy data. - Palaeogeography, Palaeoclimatology, Palaeoecology, 467: 95-119. https://doi.org/10.1016/j.palaeo.2016.05.026

Herendeen, P. S. (1992): A reevaluation of the fossil genus Podogonium Heer. - In: Herendeen, P. S., Dilcher, D. L. (eds), Advances in Legume Systematics: Part 4. The Fossil Record. Royal Botanic Gardens, Kew, pp. 3-18.

Hurník, S., Knobloch, E. (1966): Einige Ergebnisse paläontologischer und stratigraphischer Untersuchungen im Tertiär Böhmens. - Abhandlungen des Staatlichen Museums für Mineralogie und Geologie zu Dresden, 11: 17-161.

Iljinskaja [= Il'inskaya], N. (1964): Tortonskaya flora Svoshovitse [The Tortonian flora of Swoszowice]. - Trudy, Botanicheskiy Institut V. L. Komarova, Akademii Nauk SSSR, Serya 8, Paleobotanika, 5: 116-142. (in Russian)

Jechorek, H., Kovar-Eder, J. (2004): Neue Taxa aus der Flora von Weingraben (Burgenland, Miozän, Badenium). Annalen des Naturhistorischen Museums Wien, 106A: 327-343.

Jing, Zh.-zh. (1981): A mossy forest appearing in the sclerophyllous evergreen broad-leaved forests-Quercetum pannosum nimborum. - Acta Botanica Yunnanica, 3(1): 75-88.

Kordos-Szakály, M. (1984): New data to the Miocene flora of Nógrádszakál (Hungary). - Annales historico-naturales Musei nationalis hungarici, 76: 43-46.

Kottik, S. (2002): Die Palynologie des Randecker Maars; Diplomarbeit. - MS, Universität Wien, Vienna, Austria, $58 \mathrm{pp}$.

Kovar-Eder, J., Givulescu, R., Hably, L., Kvaček, Z., Mihajlovic, D., Teslenko, J., Walther, H., Zastawniak, E. (1994): Floristic changes in the areas surrounding the Paratethys during Neogene time. - In: Boulter, M. C., Fisher, H. C. (eds), Cenozoic Plants and Climates of the Arctic. NATO-ASI Series, Series I: Global Environmental Change, 27: 347-369. https://doi.org/10.1007/978-3-642-79378-3_23

Kovar-Eder, J., Jechorek, H., Kvaček, Z., Parashiv, V. (2008): The integrated plant record: an essential tool to reconstruct Neogene zonal vegetation in Europe. PALAIOS, 23: 97-111. https://doi.org/10.2110/palo.2006.p06-039r

Kovar-Eder, J., Knörr, U. C., Mazouch, P. (2012): Fruit ecology of Eocene and Neogene plant assemblages in Eu- 
rope. Tracing shifts in dispersal syndromes. - PALAIOS, 27(12): 887-903.

https://doi.org/10.2110/palo.2012.p12-051r

Kovar-Eder, J., Kvaček, Z. (2003): Towards vegetation mapping based on the fossil plant record. - Acta Universitatis Carolinae, Geologica, 46(4): 7-13.

Kovar-Eder, J., Kvaček, Z. (2007): The integrated plant record (IPR) to reconstruct Neogene vegetation - the IPR vegetation analysis. - Acta Palaeoboanica, 47(2): 391418.

Kovar-Eder, J., Kvaček, Z., Ströbitzer-Hermann, M. (2004): The Miocene Flora of Parschlug (Styria, Austria) - Revision and Synthesis. - Annalen des Naturhistorischen Museums Wien, 105A: 45-159.

Kovar-Eder, J., Schweigert, G. (in press): Revision of the plant assemblage of Steinheim am Albuch (Baden-Württemberg, Germany, reference locality of Mammal Neogene Zone MN 7). - Bulletin of Geosciences.

Kóvats, J. (1856a): Fossile Flora von Erdöbenye. - Arbeiten der geologischen Gesellschaft für Ungarn, 1: 1-37.

Kóvats, J. (1856b): Fossile Flora von Tállya. - Arbeiten der geologischen Gesellschaft für Ungarn, 1: 39-52.

Krach, W., Rutkowski, J., Kuciński, T. M. (1974): Der Sedimentationsraum und die Schichtgruppe des Saarmats s.str. in Polen. - In: Papp, A., Marinescu, F., Seneš, J. (eds), Chronostratigraphie und Neostratotypen. Miozän der Zentralen Paratethys. Bd. IV: Miozän $\mathrm{M}_{5}$, Sarmatien. Veda, Bratislava, pp. 105-112.

Kvaček, Z., Kovác, M., Kovar-Eder, J., Doláková, N., Jechorek, H., Parashiv, V., Kováčová, M., Sliva, L. (2006): Miocene evolution of landscape and vegetation in the Central Paratethys. - Geologica Carpathica, 57(4): 295-310.

Kvaček, Z., Rojík, P., Sakala, J. (2006): Tertiary of NW Bohemia. - In: Fatka, O., Kvaček, J. (eds), Excursion field guide of the 7th EPPC, Prague September 6-12, 2006. National Museum, Prague, pp. 66-75.

Łańcucka-Środoniowa, M. (1966): Tortonian flora from the "Gdów Bay" in the south of Poland. - Acta Palaeobotanica, 7: 1-135.

Łańcucka-Środoniowa, M., Zastawniak, E. (1997): The middle-Miocene flora of Wieliczka revision of Jan Zablocki's collection. - Acta Palaeobotanica, 37: 17-49.

Mantzouka, D., Kvaček, Z., Teodoridis, V., Utescher, T., Tsaparas, N., Karakitsios, V. (2015): A new late Miocene (Tortonian) flora from Gavdos Island in southernmost Greece evaluated in the context of vegetation and climate in the Eastern Mediterranean. - Neues Jahrbuch für Geologie und Paläontologie, Abhandlungen, 275(1): 47-81.

https://doi.org/10.1127/njgpa/2015/0448

Mosbrugger, V., Utescher, T. (1997): The coexistence approach - a method for quantitative reconstructions of Tertiary terrestrial palaeoclimate data using plant fossils. - Palaeogeography, Palaeoclimatology, Palaeoecology, 134: 61-86.

https://doi.org/10.1016/S0031-0182(96)00154-X

Nagymarosy, A. (1980): A magyarországi badenien korrelációja nannoplankton alapján [Correlation of the Badenian in Hungary on the basis of the nannoplankton]. Földtani Közlöny [Bulletin of the Hungarian Geological
Society], 110(2): 206-245. (in Hungarian with English summary)

Němejc, F., Kvaček, Z., Pacltová, B., Konzalová, M. (2003): Tertiary plants of the Plzeň Basin (West Bohemia). Acta Universitatis Carolinae, Geologica, 46(4/2002): 121-176.

Neubauer, T., Harzhauser, M., Kroh, A., Georgopoulou, E., Mandic, O. (2015): A gastropod-based biogeographic scheme for the European Neogene freshwater systems. - Earth-Science Reviews, 143: 98-116. https://doi.org/10.1016/j.earscirev.2015.01.010

Ou, X., Zhang, Z., Wang, Ch., Wu, Y. (2006): Vegetation Research in Meili Snow Mountain. - Science Press, Beijing, 239 pp.

Rasser, M. W., Bechly, G., Böttcher, R., Ebner, M., Grein, M., Harzhauser, M., Heizmann, E. P. J., Höltke, O., Joachim, C., Kern, A. K., Kovar-Eder, J., Nebelsick, J. H., Roth-Nebelsick, A., Schoch, R., Schweigert, G., Ziegler, R. (2013): The Miocene Randeck Maar revisited: Life in and around a peculiar volcanogenic lake. - Palaeogeography, Palaeoclimatology, Palaeoecology, 392: 426-453. https://doi.org/10.1016/j.palaeo.2013.09.025

Riederle, R., Gregor, H.-J. (1997): Die Tongrube Kirrberg bei Balzhausen - eine neue Fundstelle aus der Oberen Süßwassermolasse Bayerisch-Schwabens - Flora, Fauna, Stratigraphie. - Documenta natura, 110: 1-53.

Rüffle, L. (1963): Die obermiozäne (sarmatische) Flora vom Randecker Maar. - Paläontologische Abhandlungen, 1: 139-298.

Sachse, M., Mohr, B., Suc, J.-P. (1999): The Makrilia-flora (Crete, Greece) - A contribution to the Neogene history of the climate and vegetation of the Eastern Mediterranean. - Acta Palaeobotanica, Supplement 2: 365-372.

Sachsenhofer, R. F., Kuhlemann, J., Reischenbacher, D. (2001): Das Miozän der östlichen Norischen Senke. Arbeitstagung der Geologischen Bundesanstalt (September 3rd - 7th 2001, Neuberg an der Mürz, Austria), 2001: 135-145.

Schmid, H. (1983): Eine miozäne Blatt- und Fruchtflora von der Fossilfundstelle Sandgrube Dumerth in Burtenbach. - Heimatliche Schriftenreihe für den Landkreis Günzburg, 2: 40-46.

Schmid, W., Gregor, H.-J. (1983): Gallenbach - eine neue mittelmiozäne Fossilfundstelle in der westlichen Oberen Süßwassermolasse Bayerns. - Berichte des Naturwissenschaftlichen Vereins Schwaben, 87(3-4): 51-63.

Schmidt, C. (1976): Obermiozäne Flora von Derching bei Augsburg. - Berichte des Naturwissenschaftlichen Vereins Schwaben, "Aus der Schwäbischen Heimat", 80(34): 52-56.

Schmidt, C. (1980): Ein Profil von pflanzenführenden Schichten der Sandgrube Derching. - Berichte des Naturwissenschaftlichen Vereins Schwaben, 84(1-2): 13-15.

Schmitt, H., Butzmann, R. (1997): Entrischenbrunn. Statistische Untersuchungen an einer neuen Florenfundstelle aus der Oberen Süßwassermolasse im Landkreis Pfaffenhofen a. d. Ilm. - Documenta naturae, 110: 55-87.

Seehuber, U. (2008): Litho- und biostratigraphische Untersuchungen in der Oberen Süßwassermolasse in der Umgebung von Kirchheim in Schwabens; - InauguralDissertation zur Erlangung des Doktorgrades. - MS, 
Fakultät für Geowissenschaften, Ludwig-Maximilians-Universität München, Munic, Germany, 276 pp. + pls and tabs. (copy available on-line)

Shevenell, A. E., Kennett, J. P., Lea, D. W. (2004): Middle Miocene Southern Ocean cooling and Antarctic cryosphere expansion. - Science, 305: 1766-1770. https://doi.org/10.1126/science.1100061

Sitár, V., Kováčová-Slamková, M. (1999): Palaeobotanical and palynological study of the upper Badenian sediments from the NE part of the Vienna Basin (locality Devínska Nová Ves). - Acta Palaeobotanica, Supplement 2: 373-389.

Stur, D. (1867): Beiträge zur Flora der Süßwasserquarze, der Congerien- und Cerithienschichten im Wiener und ungarischen Becken. - Jahrbuch der kaiserlich-königlichen Geologischen Reichsanstalt, Wien, 17: 77-188.

Szafer, W. (1961): Mioceńska flora ze starych Gliwic na Śląsku [Miocene flora from Stare Gliwice in Upper Silesia]. - Prace, Instytut Geologiczny, 33: 1-205. (in Polish)

Švagrovský, J. (1978): Faziostratotypus: Devínska Nová Ves - Sandberg bei Bratislava, östlicher Rand des Wiener Beckens, Tschechoslowakei. - In: Brestenská, E. (ed.), Chronostratigraphie und Neostratotypen. Miozän der Zentralen Paratethys. Bd. VI: Miozän $\mathrm{M}_{4}$, Badenien. Veda, Bratislava, pp. 188-194.

Tang, C. Q. (2006): Evergreen sclerophyllous Quercus forests in northwestern Yunnan, China as compared to the Mediterranean evergreen Quercus forests in California, USA and northwestern Spain. - Web Ecology, 6: 88-101. https://doi.org/10.5194/we-6-88-2006

Teodoridis, V., Kovar-Eder, J., Mazouch, P. (2011a): Integrated plant record (IPR) vegetation analysis applied to modern vegetation in southeastern China and Japan. PALAIOS, 26: 623-638. https://doi.org/10.2110/palo.2010.p10-149r

Teodoridis, V., Kovar-Eder, J., Marek, P., Kvaček, Z., Mazouch, P. (2011b): The integrated plant record vegetation analysis: internet platform and online application. - Acta Musei Nationalis Pragae, Series B - Historia Naturalis, 67: 159-165.

Teodoridis, V., Kvaček, Z., Zhu, H., Mazouch, P. (2012): Environmental analysis of the mid-latitudinal European Eocene sites of plant macrofossils and their possible analogues in East Asia. - Palaeogeography, Palaeoclimatology, Palaeoecology, 333-334: 40-58. https://doi.org/10.1016/j.palaeo.2012.03.008

Unger, F. (1841-1847): Chloris protogaea. - W. Engelmann, CX, Leipzig, 150 pp.

Utescher, T. Erdei, B., François, L., Mosbrugger, V. (2007): Tree diversity in the Miocene forests of Western Eurasia. - Palaeogeography, Palaeoclimatology, Palaeoecology, 253: 226-250. https://doi.org/10.1016/j.palaeo.2007.03.041

Van Dam, J. A., Utescher, T. (2016): Plant and micromammal-based paleoprecipitation proxies: Comparing results of the Coexistence and Climate-Diversity Approach. Palaeogeography, Palaeoclimatology, Palaeoecology, 443: 18-33. https://doi.org/10.1016/j.palaeo.2015.11.010

Wu, Z. Y., Zhu, Y. C., Jiang, H. C. (1987): The Vegetfation of Yunnan. - Science Press, Beijing, 1024 pp.

Zastawniak, E. (1980): Sarmatian leaf flora from the southern margin of the Holy Cross Mts. (South Poland). Prace Muzeum Ziemi, 33: 39-107.

Zhou, Z. K., Pu, C. X., Chen, W. Y. (2003): Relationships between the distributions of Quercus sect. Heterobalanus (Fagaceae) and uplift of Himalayas. - Advances in Earth Science, 18: 884-890.

Zidianakis, G., Iliopoulos, G., Fassoulas, C. (2010): A new Late Miocene plant assemblage from Messara Basin (Crete, Greece). - Bulletin of the Geological Society of Greece, 43: 781-792. https://doi.org/10.12681/bgsg.11244

Appendix 1. Scores for the individual Middle Miocene European sites according to the IPR vegetation analysis. Numbers corresponding to those in Table 1.

Appendix 1 is available on-line on the journal website. 


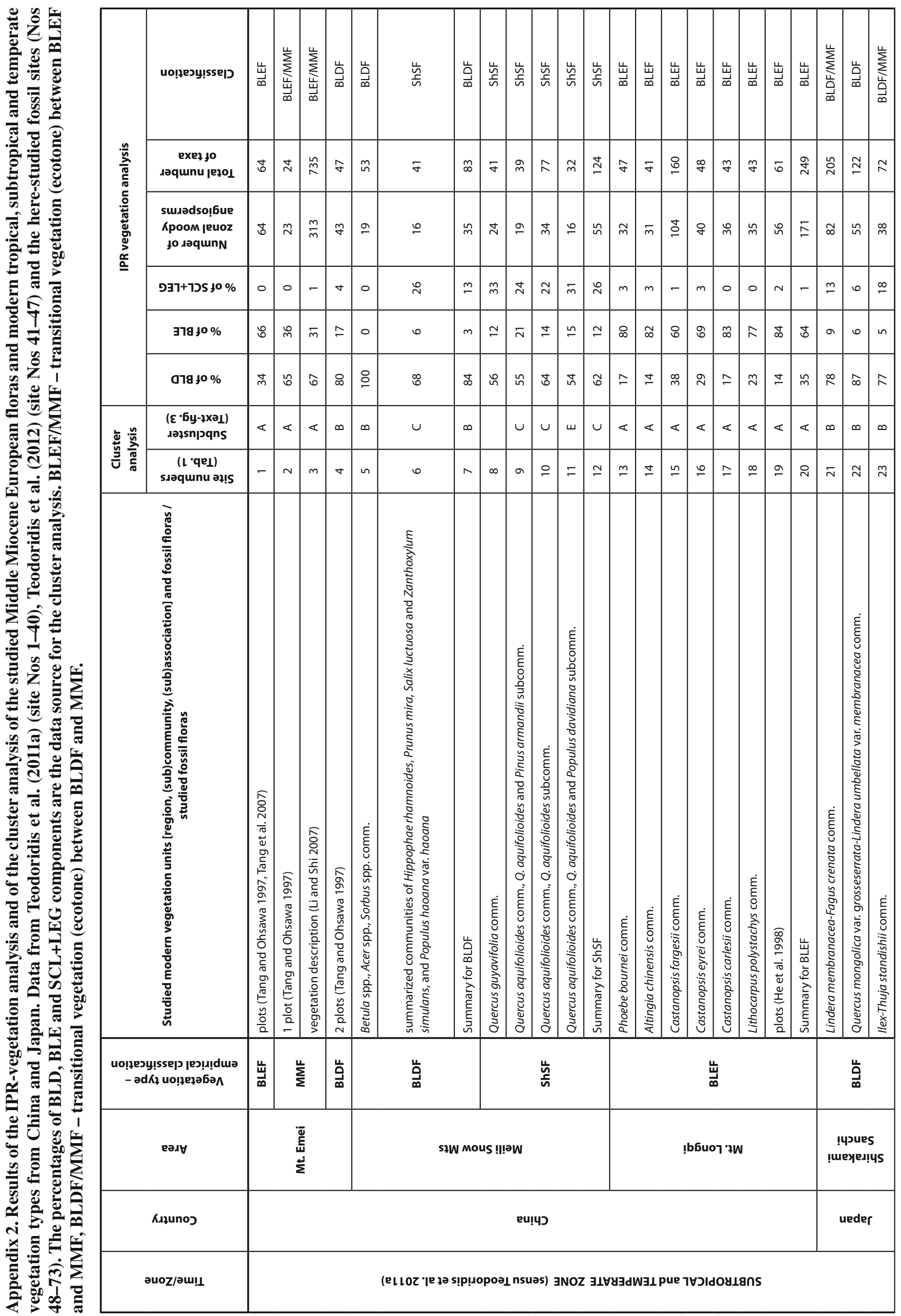




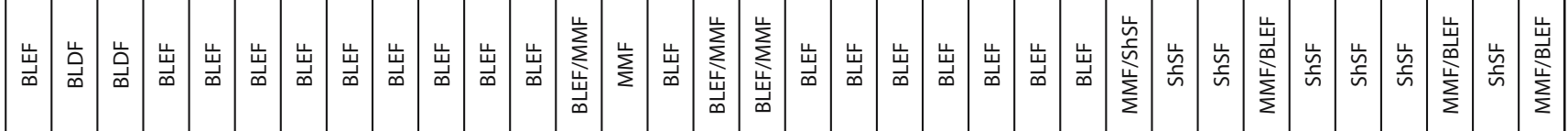

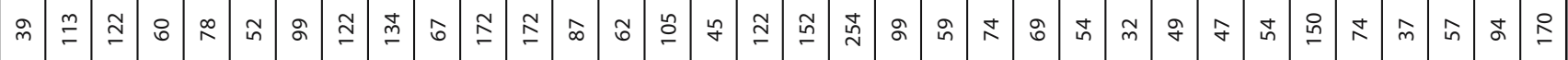

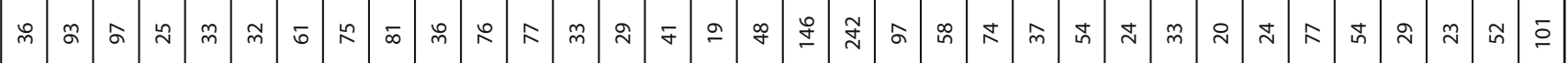

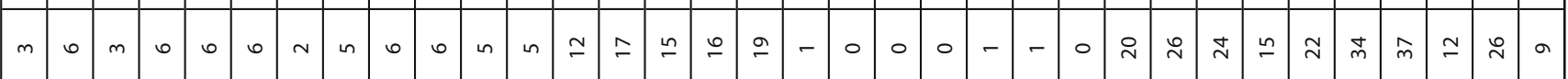

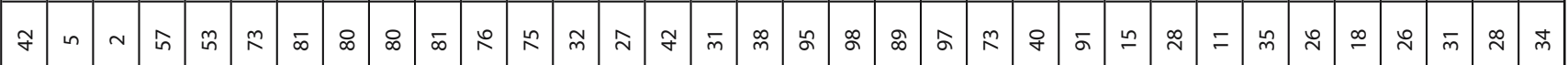

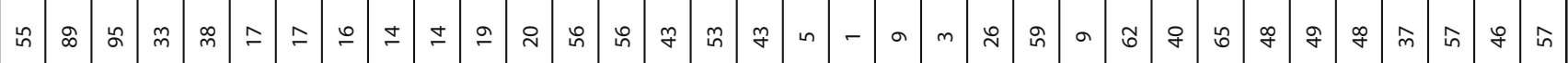

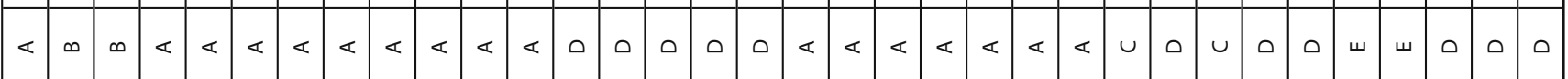

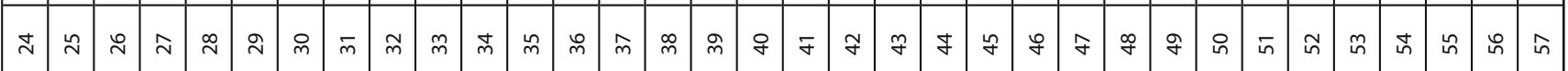

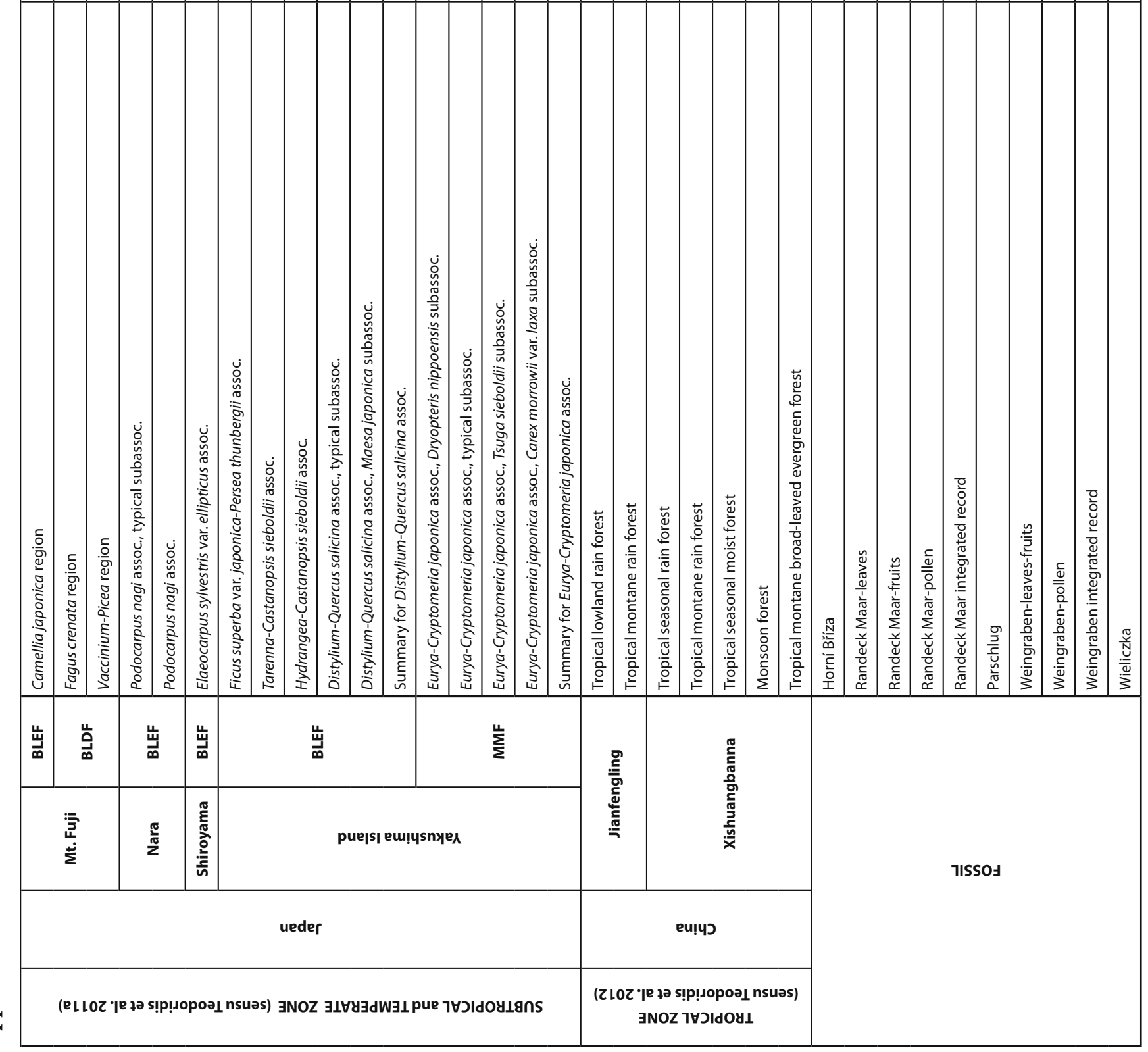




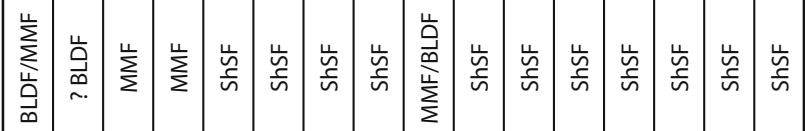

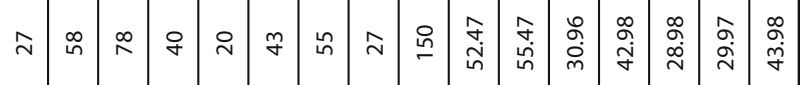

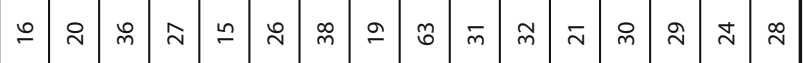

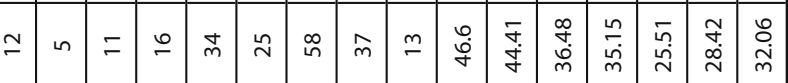

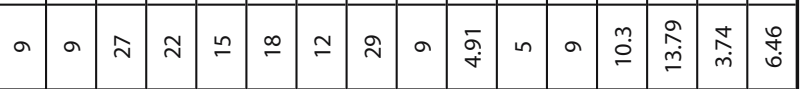

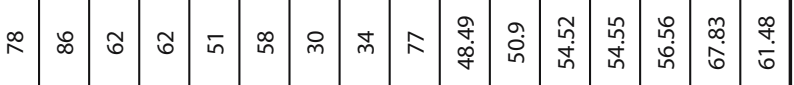

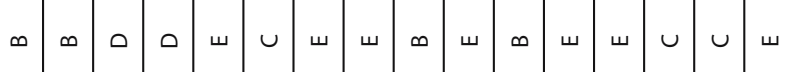

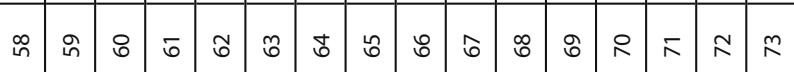

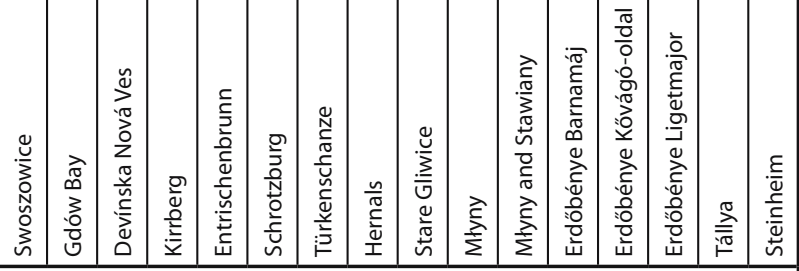

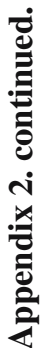

7ISSO 